\title{
CARM1 Promotes Gastric Cancer Progression by Regulating TFE3 Mediated Autophagy Enhancement via Cytoplasmic AMPK-mTOR and Nuclear CARM1- TFE3 Signaling Pathways
}

\section{Suzhen Yang}

Xi'an Jiaotong University Second Affiliated Hospital

Jing Zhang

xi'an jiaotong daxue diyi fushu yiyuan: Xi'an Jiaotong University Medical College First Affiliated Hospital

Di Chen

Air Force Medical University Xijing Hospital: Xijing Hospital

Jiayi Cao

Northwestern University Department of Medicine

\section{Ying Zheng}

Xi'an Jiaotong University Second Affiliated Hospital

\section{Yuying Han}

Northwestern University Department of Medicine

Yirong Jin

Air Force Medical University Xijing Hospital: Xijing Hospital

Shuhui Wang

Shenzhen Shekou People's Hospital

\section{Ting Wang}

Xi'an Jiaotong University Second Affiliated Hospital

\section{Lin Ma}

Shaanxi Provincial People's Hospital

\section{Tingting Luo}

Northwestern University Department of Medicine

\section{Yan Wang}

Xi'an Jiaotong University Second Affiliated Hospital

\section{Wen Qin}

Air Force Medical University School of Stomatology

Lei Dong ( $\sim$ dong556@126.com )

Xi'an Jiaotong University https://orcid.org/0000-0001-5495-7121 


\section{Primary research}

Keywords: Autophagy, CARM1, TFE3, AMPK, Gastric cancer

Posted Date: November 23rd, 2021

DOI: https://doi.org/10.21203/rs.3.rs-1088335/v1

License: (c) (i) This work is licensed under a Creative Commons Attribution 4.0 International License. Read Full License 


\section{Abstract}

Background: The role of CARM1 in tumors is contradictory. It acts as an oncogene in most kinds of cancers while inhibits the progression of liver and pancreatic cancers. CARM1 has recently been reported to regulate autophagy, which is also context-dependent. However, the effect of CARM1 on gastric cancer has not been studied. We aimed to explore whether CARM1 was involved in the progression of gastric cancer by regulating autophagy.

Methods: The clinical values of CARM1 and autophagy in gastric cancer were determined by immunohistochemistry and qRT-PCR. Transmission electron microscopy, immunofluorescence and western blotting were applied to recognize autophagy. The role of CARM1 in gastric cancer was investigated by CCK8, colony formation and flow cytometry assays in vitro and xenograft model in vivo. Immunoprecipitation assay was performed to illustrate the interaction of CARM1 and TFE3.

Results: CARM1 was upregulated in clinical GC tissues and cell lines, and higher CARM1 expression predicted worse prognosis. CARM1 enhanced GC cell proliferation, facilitated G1-S transition and inhibited ER stress-induced apoptosis by regulating autophagy. Importantly, the treatment of CARM1 inhibitor rescued the tumor-promoting effects of CARM1 both in vitro and in vivo. Furthermore, we proved CARM1 heightened TFE3 nuclear translocation to induce autophagy via cytoplasmic AMPK-mTOR and nuclear CARM1-TFE3 signaling pathways.

Conclusion: CARM1 promoted GC cell proliferation, accelerated G1-S transition and reduced ER stressinduced apoptosis by regulating autophagy. Mechanically, CARM1 triggered autophagy by facilitating TFE3 nuclear translocation via AMPK-mTOR and CARM1-TFE3 signaling pathways.

\section{Introduction}

Gastric cancer(GC) is the fifth commonly diagnosed malignant tumor and the fourth leading cause of cancer death globally(1), causing severe economic burden. Multidisciplinary treatment has become the mainstream treatment for GC patients, especially with the development of immunotherapy and targeted therapy(2). However, the number of patients benefiting from the new treatment is very limited due to individual heterogeneity. Therefore, it is very important to explore the fundamental molecular pathogenesis of GC and find new diagnostic biomarkers and therapeutic targets.

Autophagy is a self-eating process to recycle wastes of cells and maintain homeostasis through clearing longevity proteins or old organelles. These cellular contents and organelles are sequestered in twomembrane structures called autophagosomes, which are then fused with lysosomes to degrade the cargo(3). Autophagy is activated when cells encounter environmental stress, such as malnutrition, hypoxia, pathogen infection, oxidative stress, leading to adaptation or cell death depending on the intensity of the stimulus and the host (4). Autophagy is reported to exhibit crucial conflicting functions in tumor progression, including pancreatic adenocarcinoma, myeloid leukemia, gastric carcinoma, squamous cell carcinoma, sarcoma, multiple myeloma, etc(5-8). More importantly, there have been 
several clinical trials targeting autophagy in malignant glioma, non-small cell lung cancer, colon cancer, melanoma, pancreatic cancer and melanoma, implicating the promising prospects for targeting autophagy therapy(9).

Arginine methylation regulated by the protein arginine methyltransferase (PRMT) family is one of the most vital epigenetic modifications in autophagy(10). Of which coactivator-associated arginine methyltransferase-1 (CARM1), is special because it is the only enzyme capable of methylating arginine with proline sequences(11). CARM1 has two main domains, chromatin remodeling proteins and proteins possessing RNA binding activity, which play essential roles in chromatin remodeling, gene transcription, DNA packing, pre-mRNA splicing, and mRNA stability $(12,13)$. The role of CARM1 in cancers is paradoxical. It acts as an oncogene in lung, prostate, colorectal, ovarian, breast cancer, Osteosarcoma, myeloid leukemogenesis and multiple myeloma(14-23). However, CARM1 inhibits the progression of liver and pancreatic cancers $(19,20)$. Previous studies mainly focused on the role of CARM1 as a coactivator in regulating tumor-related gene $(24,25)$. Recent studies have shown that CARM1 is an important regulator of autophagy mainly through two dependent pathways. In the nucleus, AMPK is activated under glucose starvation and subsequently phosphorylates and activates FOXO3a, leading to a decrease in SKP2 expression and resulting in the stabilization of CARM1 in the nucleus, which acts as a coactivator of TFEB to promote autophagy-related gene transcription(26). While in the cytoplasm, C9orf72 mediates CARM1 lysosomal degradation by interacting with the PH-like domain and modulates lipid metabolism(27). However, the role of CARM1 in GC has not been reported previously, and it remains unclear whether CARM1 could affect the progression of GC by regulating autophagy.

In this study, we illustrate the effect of CARM1 on GC and the mechanism of CARM1 regulating autophagy. CARM1 and autophagy marker were upregulated in GC tissues and higher CARM1 expression predicted worse prognosis. Knockdown CARM1 resulted in autophagy flux blockade and succeeding ER stress-induced apoptosis, reduced cell proliferation via increased G1 phase cell cycle arrest, which could be partially reversed by autophagy agonists rapamycin. While overexpression of CARM1 exhibited the opposite results. More importantly, the treatment of CARM1 inhibitor could rescue the tumor-promoting effects of CARM1 both in vitro and in vivo. Furthermore, we proved CARM1 facilitated TFE3 nuclear translocation to induce autophagy via cytoplasmic AMPK-mTOR and nuclear CARM1-TFE3 signaling pathways. Thus, our findings suggest CARM1 alone or in combination with agents represent novel therapeutic strategies targeting GC.

\section{Methods}

\section{Tissue microarray and clinical samples}

Tissue microarray (G6038-3) including 48-paired GC tissues and relevant adjacent non-neoplastic tissues were obtained from Servicebio Biotech (Wuhan, China) containing clinical information such as gender, age, tumor location and size, metastasis, vessel carcinoma embolus and the overall survival of patients. After immunohistochemistry assay, the immunostaining intensity scores were evaluated by two 
experienced pathologists, performed double-blind reading. The percentage of positive cells and the staining intensity were analyzed by semi-quantitative results according to the following criterion: On the one hand, the percentage of positive cells was divided into 5 grades: the number of positive cells $<5 \%$ was $0,5 \% \sim 25 \%$ was $1,26 \% \sim 50 \%$ was $2,51 \% \sim 75 \%$ was $3,76 \% \sim 100 \%$ was 4 points. On the other hand, the staining intensity was recognized as 4 grades: negative was 0 , weak was 1 , moderate was 2 , and strong was 3 points. The total scores were calculated by adding up the product on a scale range of 0 12. Tissues of total score $<7$ were classified into low expression while score $\geq 7$ were recognized as high expression. 33 pairs of clinical tissues were collected from the Second Affiliated Hospital of Xi 'an Jiaotong University with informed consent from patients.

\section{Immunohistochemistry (IHC)}

Then tissue microarray and paraffin sections were soaked in xylene twice for deparaffinating. Rehydrated the slides with series ethanol (100\%, 95\%, $85 \%$ and $75 \%)$. Slides were evenly spaced in citrate buffer for microwaving treatment to perform antigen retrieval. Afterwards, added $3 \%$ hydrogen peroxide to remove endogenous peroxidase and then the slides were blocked with goat serum for 30 minutes at room temperature (RT). Then, added appropriate concentration of primary antibodies and incubated at $4{ }^{\circ} \mathrm{C}$ overnight. The next day, added secondary biotinylated antibodies and incubated at RT for 1 hour. Then, fresh DAB staining solution was added and the staining degree was observed under a microscope. Counterstained in haematoxylin bath for 30-60 seconds. Finally, dehydrated the slides in graded ethanol (75\%, $85 \%, 95 \%$ and $100 \%)$ and transferred to dimethylbenzene, and then added neutral resins to seal the slides.

\section{RNA extraction and qRT-PCR}

Total RNA was extracted by TRIzol (Invitrogen, Thermo, Waltham, MA, USA). Afterwards, the messenger RNA was transcribed reversely by the Transcriptor First-Strand cDNA Synthesis Kit (Roche, Basel, Switzerland). Subsequently, we used the FastStart Universal SYBR Green Master (Roche) to conduct qRTPCR analysis following the manufacturer's instructions. All of the primer sequences were listed in Additional file 4: Table S2.

\section{Cell culture}

Human GC cell lines HGC27, BGC823, MKN45, SNU-1 and the normal gastric epithelial cell line GES1 were purchased from Genechem (Shanghai, China), identified by STR profiling and tested free of mycoplasma contamination. All cell lines were cultured in high glucose DMEM (HyClone, Logan, Utah, USA) supplemented with 10\% fetal bovine serum (Gemini, Calabasas, CA, USA) and 1\% penicillin-streptomycin (Gibco, Grand Island, NY, USA) in a humidified incubator in $5 \% \mathrm{CO} 2$ at $37^{\circ} \mathrm{C}$.

\section{Western blotting}

Total protein was extracted using cold RIPA cracker buffer (Beyotime, Shanghai, China). Cytoplasmic and nuclear extracts were separated by applying NE-PER Nuclear and Cytoplasmic Extraction Reagents (Thermo Scientific ${ }^{\mathrm{TM}}, \mathrm{MA}, \mathrm{USA}$ ). Determine the protein concentration of each cell lysate using BCA Protein 
Assay Kit (TIANGEN, Beijing, China). Next, the same amount of protein samples was applied to SDS-PAGE gel wells and then transferred to the PVDF membranes. The membrane was incubated overnight at $4{ }^{\circ} \mathrm{C}$ in a primary antibody solution. The involved antibodies were demonstrated in Additional file 5: Table S3. The next day, the membrane was incubated with horseradish peroxidase bound secondary antibody and visualized with enhanced chemiluminescence reagents.

\section{Lentivirus and siRNA transfection}

The overexpression lentivirus and control lentivirus were obtained from Hanbio Biotrchnology (Shanghai, China). The component order of overexpression lentivirus was HBLV-h-CARM1-3xflag-ZsGreen-PURO, while the control component was HBLV-ZsGreen-PURO. The transduction was performed according to the manufacturer's protocol. Then culture the transfected cells in medium containing $2.5 \mu \mathrm{g} / \mathrm{mL}$ puromycin to obtain stable overexpression cells. HGC27 and BGC823 CARM1-overexpression cells were transfected with specific siRNAs (GenePharma, Shanghai, China), targeting CARM1 and TFE3 respectively. Lipofectamine 2000 reagent (Invitrogen, Calrsbad, CA) was added to facilitate transfection in accordance with the manufacturer's instructions. The interference sequences involved were shown in Additional file 4: Table S2.

\section{Transmission electron microscopy}

Cells were harvested and fixed at $4{ }^{\circ} \mathrm{C}$ for 2-4hours with electron microscope fixation solution (Servicebio, China) and further fixed with $1 \%$ osmium $\cdot 0.1 \mathrm{M}$ phosphate buffer for 2 hours. Dehydrated cells in graded ethanol $(50 \%, 70 \%, 80 \%, 90 \%, 95 \%$ and $100 \%)$ and $100 \%$ acetone twice. Embed and cut cells into slices of $60-80 \mathrm{~nm}$ with ultra-thin slicing machine. Then, double staining of $2 \%$ uranium acetate-lead citrate was used. At last, under transmission electron microscopy (HITACHI, Tokyo, Japan), photographs of eight random fields in every sample were captured.

\section{Immunofluorescence}

Cells were cultured on small cover glass. $4 \%$ paraformaldehyde was added into the hole for cell fixation for 30 minutes at RT. Then treat cells with $0.1 \%$ Triton 100 to permeate cells for 15 minutes. Block cells with $5 \%$ goat serum without washing. Then sufficient amount of the appropriate concentration of primary antibodies were added overnight in a moist container at $4^{\circ} \mathrm{C}$. In the following day, cells were incubated with fluorescence labeled secondary antibody and for 1 hour and DAPI for 1 minute in dark. In the end, sealed the coverslip with anti-fading buffer (BD Biosciences, NJ, USA). Photographs of five random fields were captured with confocal microscopy (Nikon C2, Tokyo, Japan).

\section{CCK8 assay}

Cell suspensions were added in 96-well plates with $3 \times 10^{3}$ cells per well in $100 \mu \mathrm{L}$ DMEM and incubated for 1-4 days. Each well was added 10ul CCK-8 regent (7 Sea Pharmatech Co., Ltd, Shanghai, China) and incubated in the cell incubator for 1 hour. Then read the absorbance value at $450 \mathrm{~nm}$ determined by a microplate spectrophotometer (Thermo, Waltham, MA, USA).

\section{Colony formation assay}


Add cells in the exponential growth phase at a density of $1 \times 10^{3}$ cells each well in 6 -well plates. Then cultivate the cells for 10-14 days until visible clones were observed. In the process, replace fresh culture medium timely according to the $\mathrm{pH}$ change. Afterwards, fixed cells with $4 \%$ paraformaldehyde for 20 minutes and stained with crystal violet dye for 10 minutes. Finally, counted the number of colonies containing more than 50 cells.

\section{Flow cytometry}

$2 \times 10^{5}$ cells were plated in 6-well plates and grew in serum-free medium for 24 hours to keep the same cell cycle. Then culture cells for another 24 hours. The adherent cells were digested and fixed by $70 \%$ ice ethanol at $4{ }^{\circ} \mathrm{C}$ overnight. The next day, the fixed cells were washed and resuspended with $500 \mu \mathrm{L}$ $\mathrm{PI} / \mathrm{RN}$ ase Staining Buffer (BD Biosciences, Franklin Lakes, NJ, USA). After culturing for 15 minutes in dark at RT, flow cytometry (BD Biosciences, Franklin Lakes, NJ, USA) was used to detect different cell cycle phases of cells.

Seed $1.5 \times 10^{5}$ cells in 6-well plates each well and then continue to cultivate cells to a confluence of $80 \%-90 \%$. Afterwards, the proportion of apoptosis cells was determined by PE Annexin V/7-aminoactinomycin (7-AAD) Detection Kit (BD Biosciences, NJ, USA) following the manufacturer's instructions and analyzed by flow cytometry.

\section{In vivo xenograft tumor model}

A subcutaneous xenograft model was established using 5-6 weeks old male BALB/c nude mice (Xi'an Jiaotong University Medical Laboratory Animal Center, Xi'an, China). The experiments were approved by the animal ethics committee of Xi'an Jiaotong University. We subcutaneously injected $1.0 \times 10^{6}$ tumor cells into the left groin of nude mice where blood flow was abundant. The volume of subcutaneous tumor was evaluated every 3 days. For the drug research, nude mice injected with CARM1 overexpression cells were randomly divided into four groups at day 6 when tumor volumes reached $50 \mathrm{~mm} 3$. CARM1 inhibitor EZM2302 was performed twice a day at $100 \mathrm{mg} / \mathrm{kg}$ i.p. HCQ was administered once a day at $50 \mathrm{mg} / \mathrm{kg}$ i.p. The combination group was given two treatments and the control group was only intraperitoneally injected with PBS. When the subcutaneous tumor showed ulceration and necrosis, the mice were sacrificed, the transplanted tumors were removed, and the weight and volume were measured.

\section{Immunoprecipitation assay}

The cells were harvested and added appropriate amount of IP lysis buffer (including protease inhibitor) for 30 min at $4^{\circ}$. After centrifugation, $6 \mu \mathrm{g}$ CARM1 antibody or control lgG and $40 \mu \mathrm{L}$ Protein A/G Mix Magnetic Beads (Merck Millipore, Germany) were added into the supernatant and incubated at $4^{\circ} \mathrm{C}$ overnight. After immunoprecipitation reaction, the protein was carefully eluted from the protein A/Gbeads and denatured for western blotting analysis.

\section{Statistical analysis}


We used GraphPad Prism 6 (San Diego, CA, USA) and SPSS 20.0 (Chicago, IL, USA) to perform statistical analysis. When comparing the differences in measurement data between the two groups, Student's $t$ test was used. When comparing the differences between more than two groups, ANOVA was applied, in which $p$ value were adjusted in face of multiple comparisons. Before Student's $t$ test and ANOVA, a variance homogeneity test and normality analysis were carried out. Overall survival curves were analyzed by the Kaplan-Meier method and log-rank tests. Chi-squared test was utilized to estimate the difference of CARM1 level between gastric cancer tissues and adjacent non-tumor tissues. The hazard ratio in clinical samples was determined by cox proportional hazards model for univariate and multivariate analyses. All values demonstrate means \pm SD. All statistical tests were two-sided, and $P<0.05$ was considered statistically significant.

\section{Results}

\section{CARM1 and autophagy marker are upregulated in human GC tissues and higher CARM1 expression demonstrates poor prognosis}

Through analysis of CARM1 expression in TCGA database, we found CARM1 mRNA expression was upregulated in GC compared to normal tissue samples (80 cases) in Fig. 1B. CARM1 expression also increased in GEPIA database (Additional file 1: Fig. S1A). To further investigate the expression of CARM1 in GC tissues, we used GC tissue microarrays containing 48 pairs of patients. The immunohistochemistry (IHC) staining indicated that CARM1 expression increased substantially in GC tissues in comparison with that in adjacent non-tumor tissues (Fig. 1A, C). Furthermore, as shown in Fig. 1D, the overall survival was shorter in patients with higher CARM1 expression, consistent with the KM Plotter database (Additional file 1: Fig. S1B), suggesting that CARM1 was an important biomarker of GC. Moreover, univariate and multivariate cox regression analyses demonstrated that CARM1 was an independent risk factor for predicting poor survival $($ Table 1,2$)$. The association of CARM1 level with clinicopathological parameters of GC patients was shown in Additional file 3: Table S1. Due to the important role of autophagy in tumor progression and the regulatory role of CARM 1 on autophagy, we analyzed the expression of autophagy markers in human GC tissues. As detailed in Fig. 1E, ATG5, LC3B and beclin1 protein expression increased in cancer compared to normal tissues, meanwhile, the mRNA levels were also higher in cancer analyzed by 33 paired clinical samples (Fig. 1F). 
Table 1

Prognostic factors in gastric cancer patients by univariate analysis

\begin{tabular}{|c|c|c|c|c|c|c|c|}
\hline \multirow[t]{2}{*}{ Parameter } & \multirow[t]{2}{*}{$\mathrm{n}$} & \multicolumn{2}{|c|}{$\begin{array}{l}\text { Cumulative survival } \\
\text { rates }(\%)\end{array}$} & \multirow{2}{*}{$\begin{array}{l}\text { Mean } \\
\text { survival } \\
\text { time } \\
\text { (month) }\end{array}$} & \multirow[t]{2}{*}{$\begin{array}{l}\text { Hazard } \\
\text { ratio }\end{array}$} & \multirow{2}{*}{$\begin{array}{l}95 \% \\
\text { Confidence } \\
\text { interval }\end{array}$} & \multirow[t]{2}{*}{$\begin{array}{l}P \\
\text { value }\end{array}$} \\
\hline & & 3-y & $5-y$ & & & & \\
\hline \multicolumn{8}{|l|}{ Gender } \\
\hline Male & 30 & 82.3 & 76.5 & 66.128 & & & \\
\hline Female & 18 & 57.2 & 57.2 & 47.510 & 2.642 & $0.883-7.905$ & 0.082 \\
\hline \multicolumn{8}{|l|}{ Age } \\
\hline$<60$ & 26 & 70.5 & 70.5 & 58.749 & & & \\
\hline$\geq 60$ & 22 & 76.5 & 69.5 & 61.513 & 0.911 & $0.306-2.716$ & 0.867 \\
\hline \multicolumn{8}{|l|}{ Location } \\
\hline Antrum & 16 & 81.3 & 81.3 & 64.961 & & & \\
\hline Cardia fundus & 22 & 70.3 & 70.3 & 58.975 & 1.768 & $0.44-7.096$ & 0.422 \\
\hline gastric body & 7 & 85.7 & 85.7 & 64.611 & 0.948 & $0.098-9.122$ & 0.963 \\
\hline Full stomach & 3 & 33.3 & 0 & 34.897 & 6.093 & $\begin{array}{l}1.216- \\
30.532\end{array}$ & 0.028 \\
\hline \multicolumn{8}{|l|}{ Tumor size } \\
\hline$<5 \mathrm{~cm}$ & 29 & 82.6 & 82.6 & 64.143 & & & \\
\hline$\geq 5 \mathrm{~cm}$ & 19 & 57.6 & 48.0 & 52.100 & 2.649 & $0.864-8.124$ & 0.088 \\
\hline \multicolumn{8}{|l|}{$\begin{array}{l}\text { Lymph node } \\
\text { metastasis }\end{array}$} \\
\hline Negative & 20 & 85.0 & 85.0 & 67.608 & & & \\
\hline Positive & 28 & 63.5 & 55.6 & 53.380 & 3.049 & $\begin{array}{l}0.835- \\
11.132\end{array}$ & 0.091 \\
\hline \multicolumn{8}{|l|}{ Metastasis } \\
\hline Negative & 44 & 78.2 & 73.9 & 62.904 & & & \\
\hline Positive & 4 & 25.0 & 25.0 & 31.048 & 3.907 & $\begin{array}{l}1.072- \\
14.234\end{array}$ & 0.039 \\
\hline \multicolumn{8}{|c|}{$\begin{array}{l}\text { Vessel carcinoma } \\
\text { embolus }\end{array}$} \\
\hline Negative & 44 & 73.1 & 68.8 & 59.570 & & & \\
\hline
\end{tabular}




\begin{tabular}{|c|c|c|c|c|c|c|c|}
\hline \multirow[t]{2}{*}{ Parameter } & \multirow[t]{2}{*}{$\mathrm{n}$} & \multicolumn{2}{|c|}{$\begin{array}{l}\text { Cumulative survival } \\
\text { rates }(\%)\end{array}$} & \multirow{2}{*}{$\begin{array}{l}\text { Mean } \\
\text { survival } \\
\text { time } \\
\text { (month) }\end{array}$} & \multirow[t]{2}{*}{$\begin{array}{l}\text { Hazard } \\
\text { ratio }\end{array}$} & \multirow{2}{*}{$\begin{array}{l}95 \% \\
\text { Confidence } \\
\text { interval }\end{array}$} & \multirow[t]{2}{*}{$\begin{array}{l}P \\
\text { value }\end{array}$} \\
\hline & & $3-y$ & $5-y$ & & & & \\
\hline Positive & 3 & 75.0 & 75.0 & 61.630 & 0.852 & $0.111-6.555$ & 0.878 \\
\hline \multicolumn{8}{|c|}{ CARM1 level } \\
\hline Low & 18 & 93.3 & 93.3 & 74.023 & & & \\
\hline High & 30 & 61.8 & 56.2 & 51.956 & 8.489 & $\begin{array}{l}1.103- \\
65.343\end{array}$ & 0.04 \\
\hline
\end{tabular}

Table 2

Multivariate analysis using the Cox proportional hazards model

\begin{tabular}{|lllll|}
\hline Parameter & $\mathbf{n}$ & Hazard ratio & $\mathbf{9 5 \%}$ Confidence interval & $P$-value \\
\hline Gender & & & & \\
\hline Male & 30 & & $0.166-4.750$ & 0.888 \\
\hline Female & 18 & 0.887 & & \\
\hline Age & & & & \\
\hline$<60$ & 26 & & $0.336-7.310$ & \\
\hline$\geq 60$ & 22 & 1.566 & & 0.068 \\
\hline Tumor size & & & & \\
\hline$<5$ cm & 29 & & & \\
\hline$\geq 5$ cm & 19 & 5.807 & & \\
\hline Metastasis & & & $0.881-38.294$ & \\
\hline Negative & 44 & & & \\
\hline Positive & 4 & 3.106 & $0.298-32.329$ & \\
\hline CARM1 level & & & & \\
\hline Low & 18 & & $1.052-63.373$ & \\
\hline High & 30 & 13.107 & & \\
\hline
\end{tabular}

\section{CARM1 promotes autophagy in GC cells}


To explore the role of CARM1 in GC cells, we evaluated the expression of CARM1 in $4 \mathrm{GC}$ cell lines and normal gastric mucosal epithelial cell line GES1. The results revealed that both CARM1 protein and mRNA expression were increased in GC cell lines compared to GES1, CARM1 levels increased slightly in BGC823 while grew dramatically in HGC27(Fig. 1G, H). Then we established CARM1-knockdown HGC27 cells using specific siRNA and stable CARM1-overexpression BGC823 cells by transfection with lentivirus. The expression of CARM1 in treated HGC27 and BGC823 cells was confirmed by western blotting and qRTPCR, presented in Fig. 2A, B.

Previous studies proved CARM1 was related to the regulation of autophagy(11), so we identified autophagosomes and autolysosomes to assess autophagy in GC cells. As can be seen in Fig. 2C, HGC27 cells with lower CARM1 expression presented fewer autophagosomes and autolysosomes in comparison with control cells, while the number in BGC823 cells transfected overexpression lentivirus increased significantly compared to normal control cells. Consistently, enhancive LC3 puncta and increased protein expression of LC3 II /I, ATG5, beclin1 and reduced p62 expression in CARM1 overexpression cells further proved that CARM1 could induce autophagy (Fig. 2D, E).

\section{Blocked autophagy flux induces endoplasmic reticulum stress}

It has been reported that autophagy and ER stress have complex interactions, and ER stress can induce autophagy through unfolded protein reactions(UPR)(28). Classical UPR includes three stress transducers, ATF6, IRE1 $a$ and PERK(29). So we evaluated ER stress related proteins and discovered that IRE1 $a$ and ATF6 expression showed no difference in GC cells treated with CARM1 siRNA or overexpression virus (Additional file 1: Fig. S1C). However, the expression of p-PERK, p-elF2 $a$ and ATF4 accelerated in CARM1 downregulation HGC27 cells while retarded when CARM1 was upregulated, consistent with the change of GRP78, a molecular chaperones of ER to help proteins fold properly(30) (Fig. 3A), suggesting knockdown of CARM1 triggered ER stress.

On the other hand, lack of autophagy can in turn promote ER stress, the inhibition of autophagy causes excess p62 accumulation, which could impair the delivery of polyubiquitinated proteins to induce UPR(31)'(32). Therefore, we utilized the autophagy agonist rapamycin to investigate whether the increased ER stress was caused by the loss of autophagy flow. As assumed, enhancive autophagy partially reverted ER stress in CARM1 downregulation cells (Fig. 3B). We further used the autophagy inhibitor HCQ to study the changes of ER stress in BGC823 cells, when autophagy was inhibited, the level of ER stress heightened (Fig. 3C). To sum up, these results showed that impaired autophagy resulted in the deposit of p62 and thereby induced ER stress.

\section{CARM1 aggravates the proliferation of GC cells through regulation of autophagy}

Since autophagy was recognized as a double-edged in the progression of cancer, we then explored the effect of CARM1 and the induced autophagy on GC cells proliferation. CCK-8 analysis revealed 
downregulation of CARM1 significantly inhibited cell growth (Fig. 4A), however, when HGC27 cells were treated with autophagy stimulant rapamycin, the decreased cell viability partially restored (Fig. 4B). On the contrary, BGC823 cells handled with autophagy suppressant HCQ attenuated cell viability induced by CARM1(Fig. 4C, D). Then we performed colony formation assay to verify the role of autophagy induced by CARM1 in cell proliferation. Similarly, upregulation of CARM1 increased colony numbers of clone, which could be reversed by HCQ (Fig. 4E, F), while downregulation of CARM1 showed the opposite effect and could be rescued by rapamycin in part (Fig. 4G, H). To further explore the role of CARM1 in tumorigenesis in vivo, we carried out a subcutaneous xenograft model in nude mice. The result showed that overexpression of CARM1 substantially accentuated tumor growth compared to mice injected with CARM1-EV cells (Fig. 4I, J). In keeping with the xenograft tumor volumes, the nude mice bearing CARM1OE cells revealed heavier weights too (Fig. 4K).

\section{CARM1 facilitates G1-S transition in cell cycle and restrains apoptosis of GC cells by inducing autophagy}

To investigate how CARM1 affect cell proliferation through regulating autophagy, we then performed cell cycle assay through flow cytometry analysis. Fig. 5A, B exhibited downregulation of CARM1 increased while upregulation decreased the proportion of cells in G0/G1 phase, accompanied by a relevant decline and promotion in $S$ phase. While treatment with rapamycin partially recovered the decrease of $S$ phase in CARM1 knockdown HGC27 cells, and HCQ reversed the increase of S phase in CARM1 overexpression cells to some extent. These results proved CARM1 accentuated G1-S transition via regulating autophagy.

Furthermore, apoptosis also played important role in the growth of tumor cells. More importantly, as shown in Fig. 3A, B, blockade of autophagy due to silencing CARM1 provoked ER stress and subsequently promoted the expression of $\mathrm{CHOP}$ and cleaved-caspase3, which were the vital proapoptotic effector(33). So we detected the apoptosis of GC cells under different treatments. As expected, the apoptosis rate enhanced in CARM1 silencing HGC27 cells, which could be counteracted by rapamycin (Fig. 5C) and in CARM1 overexpression cells presented the contrary results (Fig. 5D). Therefore, we confirmed that the inhibition of CARM1 lead to the deficient of autophagy flow, resulting in the increase of ER stress-related apoptosis.

\section{CARM1 inhibitor attenuated the tumor-promoting effect of CARM1}

Given that CARM1 could promote the GC tumor growth both in vitro and in vivo, we sought to investigate whether CARM1 inhibitor (CARM1i) EZM2302 could exert a therapeutic efficacy. As revealed in Fig. 6A, B, CARM1 $\mathrm{i}$ suppressed the increased cell viability and clone numbers caused by CARM1 overexpression. Concordantly, CARM1 $\mathrm{i}$ also triggered $\mathrm{G} 1$ phase cell cycle arrest and significantly reversed the decreased apoptosis rate in CARM1 elevated cells (Fig. 6C, D). Then we tested the treatment effect of CARM1 $\mathrm{i}$ utilizing mice xenograft models in vivo and we identified CARM1i had a beneficial therapeutic effect, 
evidenced by the slowed growth rate and eventual reduction in tumor volume, as well as the decreased tumor weight compared to the untreated group injected with CARM1 overexpression cells (Fig. 6E-G). Interestingly, CARM1i exhibited a synergistic effect in combination with the treatment of HCQ, suggesting a potential therapeutic strategy in GC (Fig. 6E-G).

\section{CARM1 activates autophagy by promoting TFE3 nuclear translocation}

Previous studies showed that the MiTF/TFE family, including TFE3, TFEB, MITF and TFEC, played important role in various physiological processes especially in lysosomal homeostasis and autophagy regulation $(34,35)$. As TFEC was mainly expressed in bone marrow-derived cells(36), we detected the MITF, TFEB and TFE3 expression to explore whether MiTF/TFE family was involved in the regulation of CARM1 on autophagy. As shown in Fig. 7A, MITF exhibited no difference while TFEB and TFE3 attenuated in CARM1 silencing cells and increased in CARM1 overexpression cells. CARM1 has been reported to interact with TFEB as its coactivator to promote transcription of autophagy-related genes(26), and TFE3 and TFEB could share regulatory networks(37). Therefore, this study mainly focused on whether CARM1 could promote autophagy via regulating TFE3. Western blotting of nuclear proteins and immunofluorescence staining demonstrated CARM1 could promote nuclear translocation of TFE3 (Fig. 7B, C). To illustrate the effect of TFE3 nuclear translocation on autophagy, we knocked down TFE3 expression using siRNA. TFE3 level was successfully silenced, bringing about the deficiency of autophagy and subsequent increased ER stress-mediated apoptotic protein expression (Fig. 7D). Functional experiments further verified TFE3 knockdown could reverse increased cell growth caused by CARM1-induced autophagy (Fig. 7E-G).

\section{The TFE3 activity is activated via cytoplasmic AMPK-mTOR and nuclear CARM1-TFE3 signaling pathways}

Then we tried to explore the mechanisms by which increased CARM1 promoted nuclear translocation of TFE3. Previous studies showed AMPK-mTOR was an important pathway involved in the regulation of MiTF/TFE family(34). Our results demonstrated in cytoplasm, p-AMPK/AMPK expression enhanced while p-mTOR/ mTOR reduced, accompanied by increased TFE3 nuclear expression in CARM1 overexpression cells (Fig. 8A), suggesting TFE3 activity was regulated by cytoplasm AMPK-mTOR signaling pathway partially. It has been reported CARM1could bind to the promoter region of TFEB to enhance transcription(26). Since TFE3 always shares regulatory mechanism with TFEB, we conducted an immunoprecipitation assay to explore whether CARM1 could also interact with TFE3, as exhibited in Fig. 8B, the overexpression of CARM1 group increased the TFE3 binding level, without the influence of different CARM1 expression levels (Fig. 8B). To further investigate the effect of AMPK on TFE3, we applied Compound $C$, an effective reversible inhibitor of AMPK(38). As shown in Fig. 8A, B, Compound C suppressed cytoplasmic AMPK-mTOR pathway and reduced both TFE3 nuclear translocation and binding activity to CARM1. 


\section{Discussion}

Gastric cancer remains one of the deadliest malignancies, lack of effective early diagnosis and prognostic molecular markers. CARM1 is elevated in various tumors and exhibits a tumor-promoting role mainly as transcriptional coactivators through methylating histones and non-histones $(25,39)$. Contradictorily, CARM1 shows tumor-inhibition effect on liver and pancreatic cancers $(19,20)$. However, the role of CARM1 in GC and involved pathomechanism have not been studied before. Here we revealed CARM1 expression increased in databases and clinical samples of GC and proved that CARM1 was an independent risk factor for predicting poor survival for the first time (Fig. 1A-D), indicating the potential detective and therapeutic strategy for GC.

Recent studies showed the vital role of CARM1 in autophagy(11). Autophagy maintains homeostasis through the degradation of long-life proteins and damaged organelles, which is considered to be a selfdefense pathway against certain stressful conditions(40). The role of autophagy in tumors is paradoxical depending on the specific environment $(41,42)$. On the one hand, autophagy markers are often located in cancer-related regions with frequent mutations or deletions as protective mechanisms to inhibit tumor initiation(43), and it has been proved that 5 -FU can inhibit GC cells by inducing autophagy related death(44).On the other hand, autophagy can help tumor cells resist nutritional deficiencies and other malignant conditions to promote tumor progression and chemotherapy resistance(45). In this study, we demonstrated that CARM1 promoted GC cells proliferation through autophagy regulated G1-S transition (Fig. 4A-H, 5A-B).

Furthermore, our results showed deficient autophagy flux caused by CARM1 inhibition contributed to ER stress related apoptosis, which affecting tumor growth too. There are three main UPR signaling pathways initiated by IRE1a, PERK, and ATF6 to lessen protein load and enhance protein-folding capacity(46), and we found the ER stress induced by CARM1 mediated autophagy was regulated mainly by PERK pathway (Fig. 3B,C). PERK undergoes dimerization and autophosphorylation, leading to activation of its kinase domain, which phosphorylates elF2a, and p-elF2a inhibits most protein translation to relieve the unfolded protein burden of ER(47) but selectively enhances ATF4 translation, leading to the increase of downstream protein $\mathrm{CHOP}$ expression(48). CHOP is responsible for the apoptosis of cells with dysfunctional ER(49). In our study, increased ER stress mediated by impaired autophagy promoted CHOP expression and eventually potentiated apoptosis (Fig. 5C).

Our results elucidated CARM1 exerted significant tumor-promoting role in GC, so we sought to explore the therapeutic effect of targeting CARM1 through utilizing small molecule compound EZM2302, which was a specific inhibitor of CARM1 and had shown significant inhibition effect on Multiple Myeloma both in vitro and in vivo(21). In AML and diffuse large B-cell lymphoma mice model, CARM1i also exhibited effective inhibitory effect $(50,51)$. We then wanted to know whether CARM1i could also inhibit the progression of GC, a solid tumor. As shown in Fig. 6A-D, CARM1i retarded GC cell viability, induced G1 cell cycle arrest and trigged apoptosis in vitro, and the implanted tumors in vivo were also restrained (Fig. 6E$\mathrm{G})$. Furthermore, the autophagy inhibitor HCQ, which had been proved to possess appreciable antitumor 
activity in clinical trials $(52,53)$, demonstrated synergistic antitumor effects with CARM1i, suggesting the combination of CARM1 $i$ and HCQ as a potential therapy target.

Lastly, we aimed to clarify how CARM1 regulated autophagy. The MiT/TFE family was reported to exert essential effect on autophagy regulation(54), we found TFEB and TFE3 both increased in CARM1 overexpression cells (Fig. 7A), as TFEB had been clearly demonstrated to interact with CARM1 to promote autophagy(26), we focused our study on whether CARM1 could induce autophagy by regulating TFE3. TFE3 helps maintain lysosomal homeostasis and promotes autolysosome formation through binding CLEAR elements of lysosome and autophagy relevant genes(55). The results showed CARM1 promoted TFE3 nuclear translocation to activate autophagy, and silencing TFE3 could reverse increased cell proliferation and decreased apoptosis induced by CARM1-mediated autophagy. As reported, MTOR complex 1 (MTORC1) was the most important regulator of TFE3 by directly phosphorylating TFE3 serine residue 321, causing TFE3 cytoplasmic retention(56). The MTORC1 activity is negatively regulated by AMPK, an energy sensor, through direct and indirect phosphorylation under energy deficiency $(57,58)$. Moreover, activated AMPK could directly phosphorylate TFE3 serine residues, resulting in TFE3 transcriptional activity(59). So we detected cytoplasmic AMPK-mTOR pathway and found activated AMPK-mTOR pathway was responsible for the nucleus translocation of TFE3(Fig. 8A). Furthermore, CARM1 was reported to coactive TFEB in the nucleus, while TFEB and TFE3 always shared regulatory network, we then explored the interaction of CARM1 and TFE3 in the nucleus and proved CARM1 could also regulate autophagy though nucleus CARM1-TFE3 signaling pathway (Fig. 8B). Interesting, a recent study reported CARM1 activated autophagy as an enhancer through CARM1-Pontin-FOXO3a signaling axis, different from the direct interaction with TFEB or TFE3(59). CARM1 appears to regulate autophagy through several functionally redundant transcription factors, suggesting the key role of CARM1 in autophagy regulation.

In conclusion, the present study first revealed CARM1 was upregulated in clinical GC tissues and cell lines, and higher CARM1 expression was related to an unfavorable prognosis. More importantly, our study proved that the treatment of CARM1i exerted significant inhibitory effect on GC tumor growth both in vivo and in vitro and showed a synergistic impact with autophagy inhibitor for the first time, suggesting a promising therapeutic target. CARM1 promoted GC cell proliferation, accelerated G1-S transition and reduced ER stress-induced apoptosis by regulating autophagy. Mechanically, CARM1 potentiated autophagy by facilitating TFE3 nuclear translocation and activity via activating cytosol AMPK-mTOR and nuclear CARM1-TFE3 signaling pathways.

\section{Abbreviations}

GC: Gastric cancer; CARM1: Coactivator-associated arginine methyltransferase-1; IHC:

Immunohistochemistry; DMEM: Dulbecco's modified Eagle's medium; ER stress: Endoplasmic reticulum stress; MITF: Melanocyte inducing transcription factor; TFE3: Transcription factor binding to IGHM enhancer 3; TFEB: Transcription factor EB. 


\section{Declarations}

\section{Acknowledgements}

We gratefully acknowledge all supports from participants in this research.

\section{Authors' contributions}

SZY, JZ, DC and JYC carried out the experimental, collected and analyzed the data, assembled the figures and wrote the initial article. YZ, YYH, YRJ and SHW helped perform the experiments and generated data. TW, LM, TTL contributed to clinical sample collection. YW, WQ, LD designed experiments, provided key support and revised the manuscript. All authors read and approved the final manuscript.

\section{Funding}

The study was funded by Central Government Guide the Development of Local Science and Technology Special Funds (2016ZY-HM-01), Shanxi Provincial Science \& Technology Plan Project (2016KTZDSF0202), National Natural Science Foundation of China (No.82001079), and Institutional Foundation Of The First Affiliated Hospital Of Xi'an Jiaotong University (2020QN-26).

\section{Availability of data and materials}

The data and materials supporting the findings of the research are available from the corresponding author.

\section{Ethics approval and consent to participate}

The experiments were approved by the Animal Ethics Committee of Xi'an Jiaotong University. All animal experiments follow the guidelines stipulated by the Medical School of Xi'an Jiaotong University (G-222).

\section{Consent for publication}

Not applicable.

\section{Competing interests}


The authors declare no competing interests.

\section{Author details}

${ }^{1}$ Department of Digestive Disease and Gastrointestinal Motility Research Room, The Second Affiliated Hospital of Xi'an Jiaotong University, Xi'an, China. ${ }^{2}$ Department of Kidney Transplantation, Nephropathy Hospital, The First Affiliated Hospital of Xi'an Jiaotong University, Xi'an, 710061, China. ${ }^{3}$ State key Laboratory of Cancer Biology, National Clinical Research Center for Digestive Diseases and Xijing Hospital of Digestive Diseases, Air Force Military Medical University, Xi'an 710032, China. ${ }^{4}$ Faculty of Life Science, Northwest University, 229 Taibai North Road, Xi'an, Shaanxi Province, 710069. ${ }^{5}$ Department of Infectious Diseases, Shenzhen Shekou People's Hospital, Shenzhen, MI 518067, P.R. China. ${ }^{6}$ Shaanxi Provincial People's Hospital, Xi'an 710043, Shaanxi, China. ${ }^{7}$ State Key Laboratory of Military Stomatology \& National Clinical Research Center for Oral Diseases \& Shaanxi Clinical Research Center for Oral Diseases, Department of Orthodontics, School of Stomatology, Fourth Military Medical University, Xi'an, 710032, China.

\section{References}

1. Sung H, Ferlay J, Siegel RL, Laversanne M, Soerjomataram I, Jemal A, et al. Global Cancer Statistics 2020: GLOBOCAN Estimates of Incidence and Mortality Worldwide for 36 Cancers in 185 Countries. CA: a cancer journal for clinicians. 2021;71(3):209-49.

2. Joshi SS, Badgwell BD. Current treatment and recent progress in gastric cancer. CA: a cancer journal for clinicians. 2021;71(3):264-79.

3. Yang ZF, Klionsky DJ. Mammalian autophagy: core molecular machinery and signaling regulation. Current Opinion in Cell Biology. 2010;22(2):124-31.

4. He CC, Klionsky DJ. Regulation Mechanisms and Signaling Pathways of Autophagy. Annu Rev Genet. 2009;43:67-93.

5. Altman JK, Szilard A, Goussetis DJ, Sassano A, Colamonici M, Gounaris E, et al. Autophagy Is a Survival Mechanism of Acute Myelogenous Leukemia Precursors during Dual mTORC2/mTORC1 Targeting. Clinical Cancer Research. 2014;20(9):2400-9

6. Kun Z, Hanqing G, Hailing T, Yuan Y, Jun Z, Lingxia Z, et al. Gastrin Enhances Autophagy and Promotes Gastric Carcinoma Proliferation via Inducing AMPKa. Oncology research. 2017;25(8):1399-407.

7. Masui A, Hamada M, Kameyama H, Wakabayashi K, Takasu A, Imai T, et al. Autophagy as a Survival Mechanism for Squamous Cell Carcinoma Cells in Endonuclease G-Mediated Apoptosis. PloS one. 2016;11(9):e0162786. 
8. Wang Y, Xiong H, Liu D, Hill C, Ertay A, Li J, et al. Autophagy inhibition specifically promotes epithelial-mesenchymal transition and invasion in RAS-mutated cancer cells. Autophagy. 2019;15(5):886-99.

9. Onorati AV, Dyczynski M, Ojha R, Amaravadi RK. Targeting autophagy in cancer. Cancer. 2018;124(16):3307-18.

10. Baek SH, Kim KI. Epigenetic Control of Autophagy: Nuclear Events Gain More Attention. Molecular cell. 2017;65(5):781-5.

11. Suresh S, Huard S, Dubois T. CARM1/PRMT4: Making Its Mark beyond Its Function as a Transcriptional Coactivator. Trends in cell biology. 2021;31(5):402-17.

12. Bedford MT, Clarke SG. Protein arginine methylation in mammals: who, what, and why. Molecular cell. 2009;33(1):1-13.

13. Kim JH, Yoo BC, Yang WS, Kim E, Hong S, Cho JY. The Role of Protein Arginine Methyltransferases in Inflammatory Responses. Mediators Inflamm. 2016;2016:4028353.

14. Al-Dhaheri M, Wu J, Skliris GP, Li J, Higashimato K, Wang Y, et al. CARM1 is an important determinant of ERalpha-dependent breast cancer cell differentiation and proliferation in breast cancer cells. Cancer Res. 2011;71(6):2118-28.

15. Elakoum R, Gauchotte G, Oussalah A, Wissler MP, Clement-Duchene C, Vignaud JM, et al. CARM1 and PRMT1 are dysregulated in lung cancer without hierarchical features. Biochimie. 2014;97:210-8.

16. Karakashev S, Zhu H, Wu S, Yokoyama Y, Bitler BG, Park PH, et al. CARM1-expressing ovarian cancer depends on the histone methyltransferase EZH2 activity. Nat Commun. 2018;9(1):631.

17. Majumder S, Liu Y, Ford OH, 3rd, Mohler JL, Whang YE. Involvement of arginine methyltransferase CARM1 in androgen receptor function and prostate cancer cell viability. Prostate. 2006;66(12):1292301.

18. Ou CY, LaBonte MJ, Manegold PC, So AY, lanculescu I, Gerke DS, et al. A coactivator role of CARM1 in the dysregulation of beta-catenin activity in colorectal cancer cell growth and gene expression. Mol Cancer Res. 2011;9(5):660-70.

19. Wang YP, Zhou W, Wang J, Huang X, Zuo Y, Wang TS, et al. Arginine Methylation of MDH1 by CARM1 Inhibits Glutamine Metabolism and Suppresses Pancreatic Cancer. Molecular cell. 2016;64(4):67387.

20. Zhong XY, Yuan XM, Xu YY, Yin M, Yan WW, Zou SW, et al. CARM1 Methylates GAPDH to Regulate Glucose Metabolism and Is Suppressed in Liver Cancer. Cell reports. 2018;24(12):3207-23.

21. Drew AE, Moradei O, Jacques SL, Rioux N, Boriack-Sjodin AP, Allain C, et al. Identification of a CARM1 Inhibitor with Potent In Vitro and In Vivo Activity in Preclinical Models of Multiple Myeloma. Sci Rep. 2017;7(1):17993.

22. Greenblatt SM, Man N, Hamard PJ, Asai T, Karl D, Martinez C, et al. CARM1 Is Essential for Myeloid Leukemogenesis but Dispensable for Normal Hematopoiesis. Cancer Cell. 2019;35(1):156. 
23. Li S, Cheng D, Zhu B, Yang Q. The Overexpression of CARM1 Promotes Human Osteosarcoma Cell Proliferation through the pGSK3beta/beta-Catenin/cyclinD1 Signaling Pathway. International journal of biological sciences. 2017;13(8):976-84.

24. Guccione E, Richard S. The regulation, functions and clinical relevance of arginine methylation. Nat Rev Mol Cell Biol. 2019;20(10):642-57.

25. Jarrold J, Davies CC. PRMTs and Arginine Methylation: Cancer's Best-Kept Secret? Trends in molecular medicine. 2019;25(11):993-1009.

26. Shin HJ, Kim H, Oh S, Lee JG, Kee M, Ko HJ, et al. AMPK-SKP2-CARM1 signalling cascade in transcriptional regulation of autophagy. Nature. 2016;534(7608):553-7.

27. Liu Y, Wang T, Ji YJ, Johnson K, Liu H, Johnson K, et al. A C9orf72-CARM1 axis regulates lipid metabolism under glucose starvation-induced nutrient stress. Genes \& development. 2018;32(2122):1380-97.

28. Ogata M, Hino S, Saito A, Morikawa K, Kondo S, Kanemoto S, et al. Autophagy is activated for cell survival after endoplasmic reticulum stress. Molecular and cellular biology. 2006;26(24):9220-31.

29. Urra H, Dufey E, Avril T, Chevet E, Hetz C. Endoplasmic Reticulum Stress and the Hallmarks of Cancer. Trends in cancer. 2016;2(5):252-62.

30. Hendershot LM, Valentine VA, Lee AS, Morris SW, Shapiro DN. Localization of the gene encoding human BiP/GRP78, the endoplasmic reticulum cognate of the HSP70 family, to chromosome 9q34. Genomics. 1994;20(2):281-4.

31. Wang XJ, Yu J, Wong SH, Cheng AS, Chan FK, Ng SS, et al. A novel crosstalk between two major protein degradation systems: regulation of proteasomal activity by autophagy. Autophagy. 2013;9(10):1500-8.

32. Pankiv S, Clausen TH, Lamark T, Brech A, Bruun JA, Outzen H, et al. p62/SQSTM1 binds directly to Atg8/LC3 to facilitate degradation of ubiquitinated protein aggregates by autophagy. The Journal of biological chemistry. 2007;282(33):24131-45.

33. McComb S, Chan PK, Guinot A, Hartmannsdottir H, Jenni S, Dobay MP, et al. Efficient apoptosis requires feedback amplification of upstream apoptotic signals by effector caspase-3 or -7 . Science advances. 2019;5(7):eaau9433.

34. Slade L, Pulinilkunnil T. The MiTF/TFE Family of Transcription Factors: Master Regulators of Organelle Signaling, Metabolism, and Stress Adaptation. Mol Cancer Res. 2017;15(12):1637-43.

35. Perera RM, Stoykova S, Nicolay BN, Ross KN, Fitamant J, Boukhali M, et al. Transcriptional control of autophagy-lysosome function drives pancreatic cancer metabolism. Nature. 2015;524(7565):361-5.

36. Rehli M, Lichanska A, Cassady Al, Ostrowski MC, Hume DA. TFEC is a macrophage-restricted member of the microphthalmia-TFE subfamily of basic helix-loop-helix leucine zipper transcription factors. Journal of immunology (Baltimore, Md: 1950). 1999;162(3):1559-65.

37. Martina JA, Puertollano R. TFEB and TFE3: The art of multi-tasking under stress conditions. Transcription. 2017;8(1):48-54. 
38. Abdulrahman RM, Boon MR, Sips HC, Guigas B, Rensen PC, Smit JW, et al. Impact of Metformin and compound $\mathrm{C}$ on NIS expression and iodine uptake in vitro and in vivo: a role for CRE in AMPK modulation of thyroid function. Thyroid: official journal of the American Thyroid Association. 2014;24(1):78-87.

39. Bedford MT, Richard S. Arginine methylation an emerging regulator of protein function. Molecular cell. 2005;18(3):263-72.

40. Hale AN, Ledbetter DJ, Gawriluk TR, Rucker EB, 3rd. Autophagy: regulation and role in development. Autophagy. 2013;9(7):951-72.

41. Mathew R, Karp CM, Beaudoin B, Vuong N, Chen GH, Chen HY, et al. Autophagy Suppresses Tumorigenesis through Elimination of p62. Cell. 2009;137(6):1062-75.

42. White E. The role for autophagy in cancer. Journal of Clinical Investigation. 2015;125(1):42-6.

43. Aita VM, Liang XH, Murty VV, Pincus DL, Yu W, Cayanis E, et al. Cloning and genomic organization of beclin 1, a candidate tumor suppressor gene on chromosome 17q21. Genomics. 1999;59(1):59-65.

44. Yang C, Pan Y. Fluorouracil induces autophagy-related gastric carcinoma cell death through Beclin-1 upregulation by miR-30 suppression. Tumour biology: the journal of the International Society for Oncodevelopmental Biology and Medicine. 2015.

45. Cao Y, Luo Y, Zou J, Ouyang J, Cai Z, Zeng X, et al. Autophagy and its role in gastric cancer. Clinica chimica acta; international journal of clinical chemistry. 2019;489:10-20.

46. Hetz C, Papa FR. The Unfolded Protein Response and Cell Fate Control. Molecular cell. 2018;69(2):169-81.

47. Clarke HJ, Chambers JE, Liniker E, Marciniak SJ. Endoplasmic reticulum stress in malignancy. Cancer Cell. 2014;25(5):563-73.

48. Harding HP, Novoa I, Zhang Y, Zeng H, Wek R, Schapira M, et al. Regulated translation initiation controls stress-induced gene expression in mammalian cells. Molecular cell. 2000;6(5):1099-108.

49. Zinszner H, Kuroda M, Wang X, Batchvarova N, Lightfoot RT, Remotti H, et al. CHOP is implicated in programmed cell death in response to impaired function of the endoplasmic reticulum. Genes \& development. 1998;12(7):982-95.

50. Greenblatt SM, Man N, Hamard PJ, Asai T, Karl D, Martinez C, et al. CARM1 Is Essential for Myeloid Leukemogenesis but Dispensable for Normal Hematopoiesis. Cancer Cell. 2018;33(6):1111-27.e5.

51. Veazey KJ, Cheng D, Lin K, Villarreal OD, Gao G, Perez-Oquendo M, et al. CARM1 inhibition reduces histone acetyltransferase activity causing synthetic lethality in CREBBP/EP300-mutated lymphomas. Leukemia. 2020;34(12):3269-85.

52. Rojas-Puentes LL, Gonzalez-Pinedo M, Crismatt A, Ortega-Gomez A, Gamboa-Vignolle C, NuñezGomez R, et al. Phase II randomized, double-blind, placebo-controlled study of whole-brain irradiation with concomitant chloroquine for brain metastases. Radiation oncology (London, England). 2013;8:209. 
53. Dong X, Wang Y, Zhou Y, Wen J, Wang S, Shen L. Aquaporin 3 facilitates chemoresistance in gastric cancer cells to cisplatin via autophagy. Cell death discovery. 2016;2:16087.

54. Palmieri M, Impey S, Kang H, di Ronza A, Pelz C, Sardiello M, et al. Characterization of the CLEAR network reveals an integrated control of cellular clearance pathways. Human molecular genetics. 2011;20(19):3852-66.

55. Lim JA, Meena NK, Raben N. Pros and cons of different ways to address dysfunctional autophagy in Pompe disease. Annals of translational medicine. 2019;7(13):279.

56. Shimobayashi M, Hall MN. Making new contacts: the mTOR network in metabolism and signalling crosstalk. Nat Rev Mol Cell Biol. 2014;15(3):155-62.

57. Inoki K, Ouyang H, Zhu T, Lindvall C, Wang Y, Zhang X, et al. TSC2 integrates Wnt and energy signals via a coordinated phosphorylation by AMPK and GSK3 to regulate cell growth. Cell. 2006;126(5):955-68.

58. Gwinn DM, Shackelford DB, Egan DF, Mihaylova MM, Mery A, Vasquez DS, et al. AMPK phosphorylation of raptor mediates a metabolic checkpoint. Molecular cell. 2008;30(2):214-26.

59. Paquette M, El-Houjeiri L, L CZ, Puustinen P, Blanchette P, Jeong H, et al. AMPK-dependent phosphorylation is required for transcriptional activation of TFEB and TFE3. Autophagy. 2021:1-19.

\section{Figures}


(A)

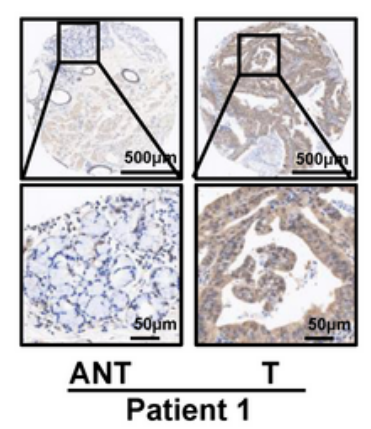

(B)

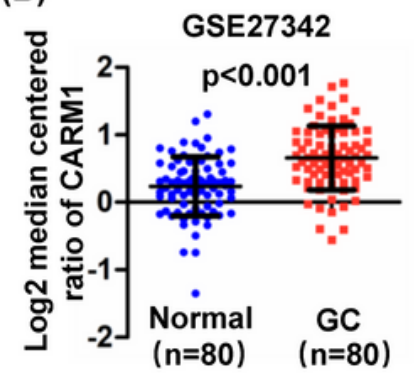

(E)

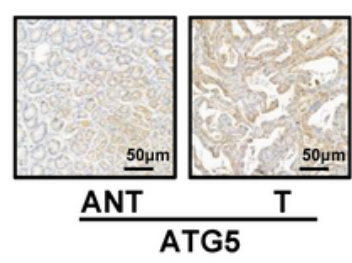

(F)

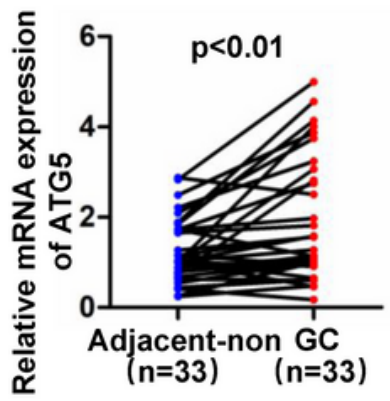

(G)

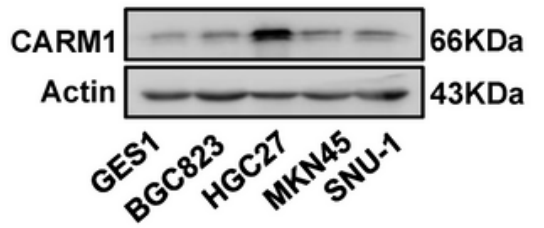

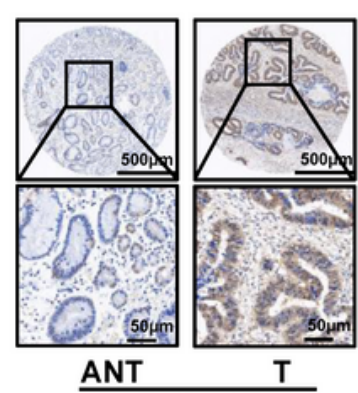

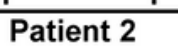

(C)
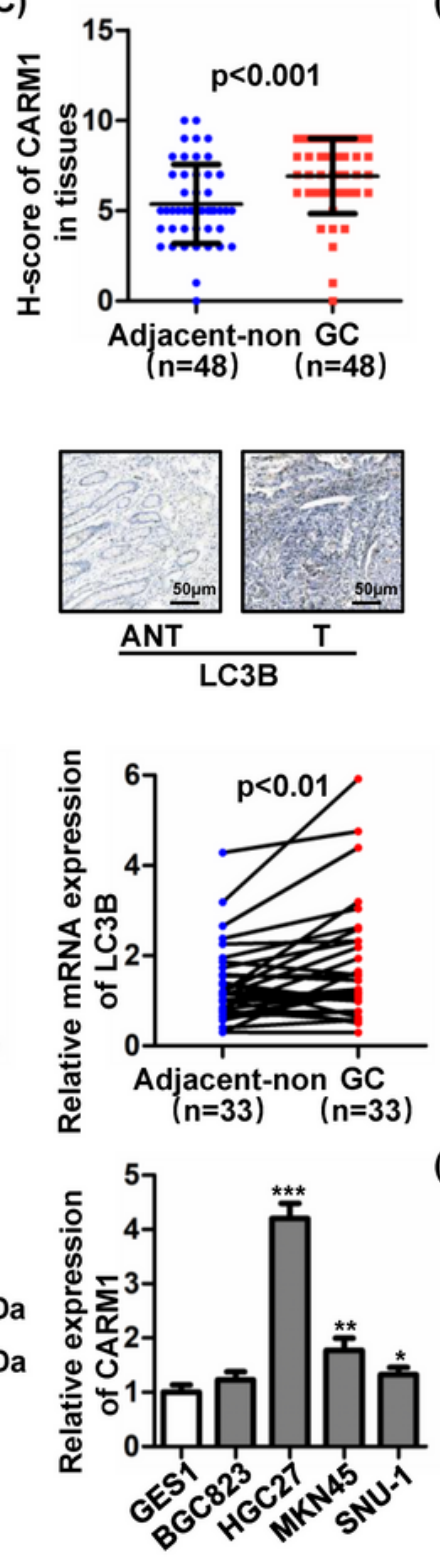

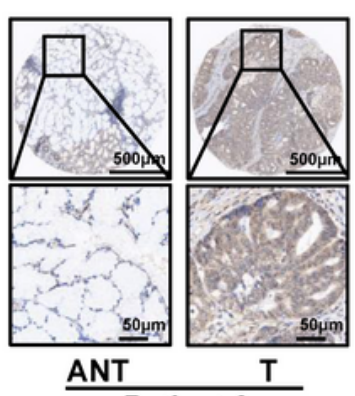

Patient 3

(D)
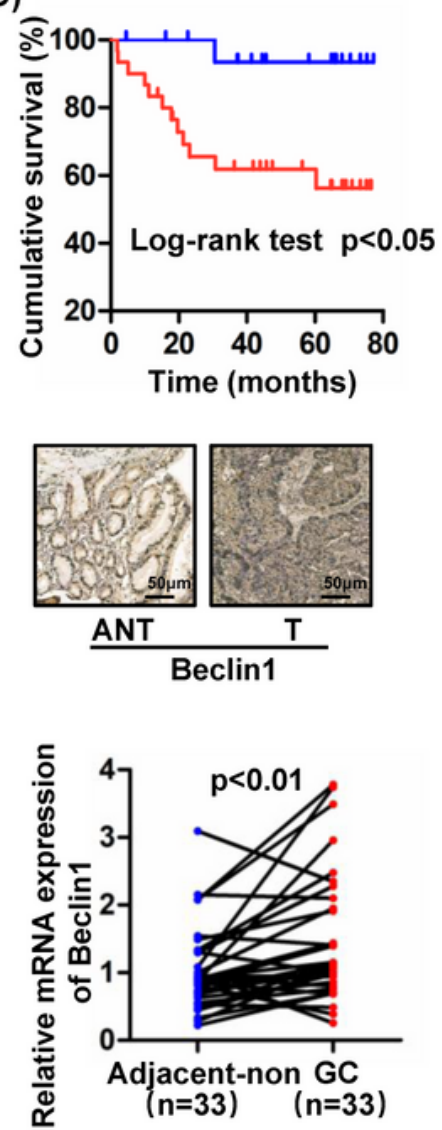

(H)

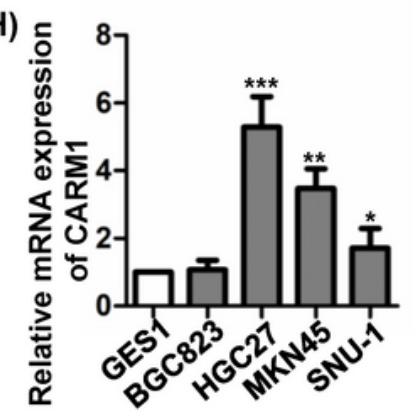

\section{Figure 1}

CARM1 and autophagy marker increase in GC tissues and higher CARM1 expression predicts worse prognosis. A Representative images of immunohistochemistry (IHC) staining of CARM1 expression in 48

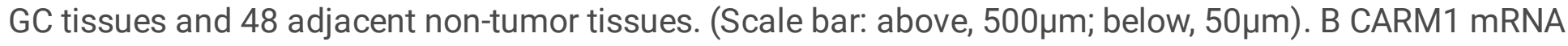
expression was significantly upregulated in GC tissues $(n=80)$ compared to normal tissues $(n=80)$ in the TCGA profile (Student's t test, P < 0.001).C H-score of CARM1 in GC tissues and adjacent non-tumor 
tissues $(n=80)$ were evaluated by the staining intensity. (Student's $t$ test, $P<0.001)$. D Kaplan-Meier curve depicting the overall survival of GC patients $(n=48)$ stratified by high and low expression of CARM1 (Logrank test, $P$ <0.05). E Representative photographs of IHC staining of autophagy marker ATG5, LC3B, Beclin1 in 33 pairs of GC tissues and adjacent non-tumor tissues. (Scale bar: $50 \mu \mathrm{m}$ ). F The mRNA expression of ATG5, LC3B, Beclin1 of 33 pairs of cancer and adjacent tissues was evaluated. G Western blot analysis of CARM1 protein expression in human GC cell lines and gastric mucosal epithelial cell line. $\beta$ - Actin was used as a loading control. H mRNA expression levels of CARM1 in four human GC cell lines and GES-1 cell line were determined by qRT-PCR. Bars represent mean \pm SD from three independent experiments. *Represents Student's t test ${ }^{*} P<0.05,{ }^{\star} \mathrm{P}<0.01$ and ${ }^{\star *} * \mathrm{P}<0.001$. 
(A)

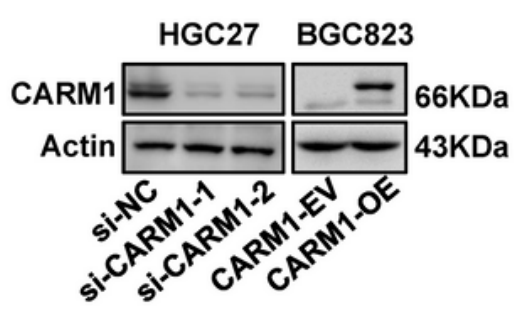

(C)

HGC27

$1500 \times$

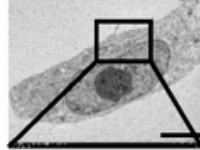

$6000 x$
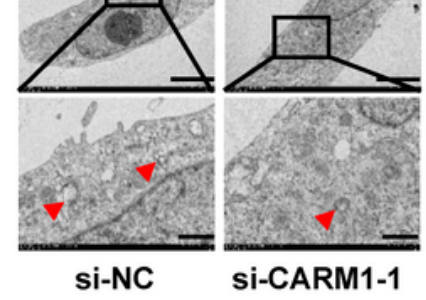

si-CARM1-1

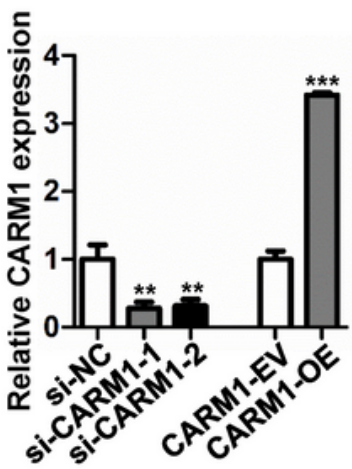

(B)

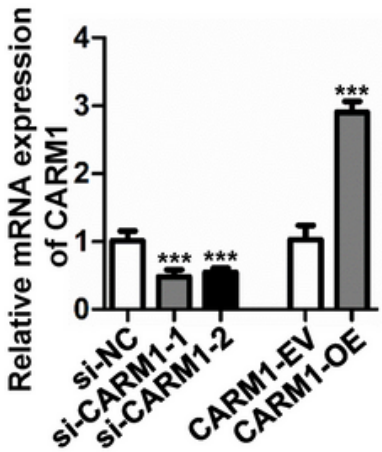

BGC823

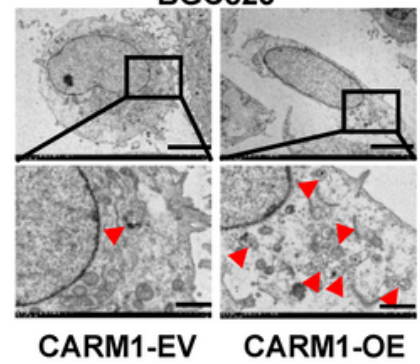

(D)
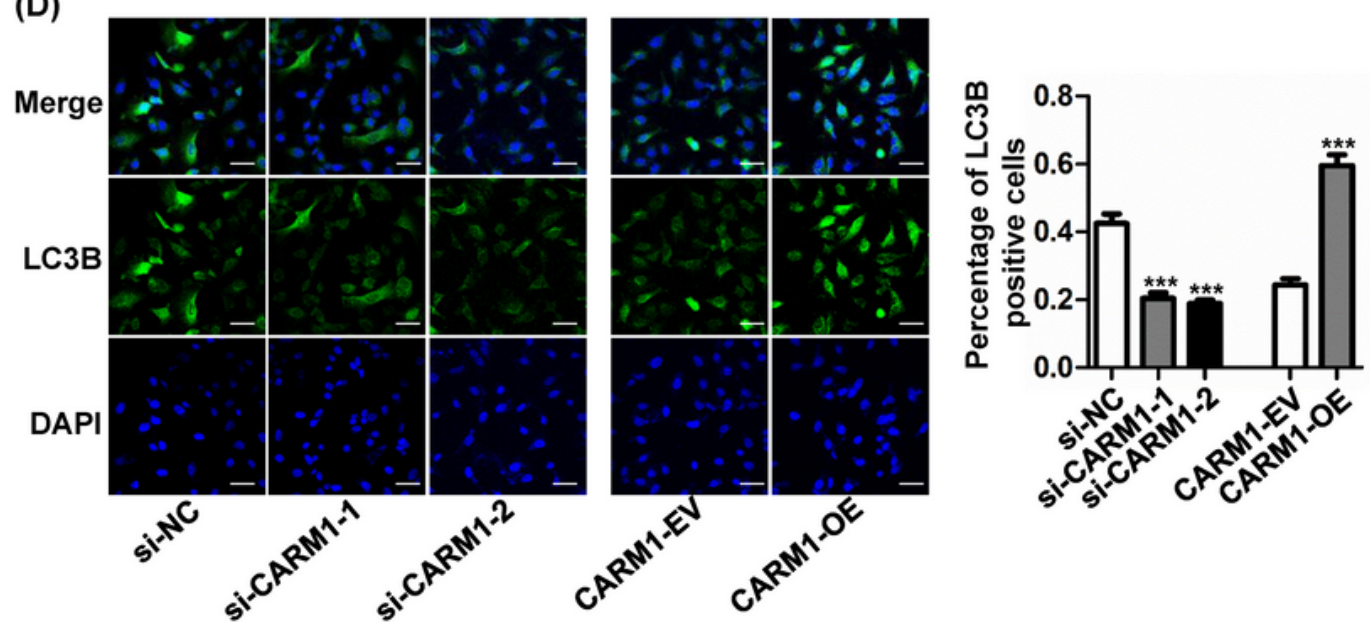

(E)
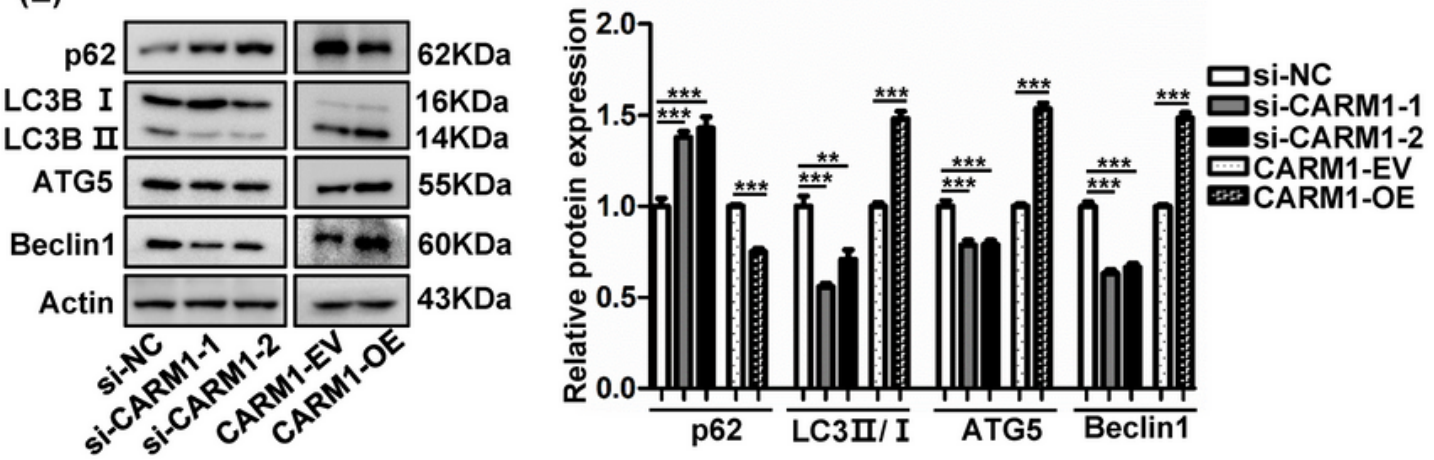

Figure 2

CARM1 enhances autophagy of GC cells. A and B Knockdown of CARM1 in HGC27 cells and overexpression of CARM1 in BGC823 cells effects were confirmed by western blotting and qRT-PCR. C Representative images of autophagosome and autolysosome (indicated by the red arrows) were obtained from transmission electron microscopy. The images above indicated lower magnification (scale bar: $5 \mu \mathrm{m})$ and the images below indicated higher magnification (scale bar: $1 \mu \mathrm{m})$. D Representative 

levels of p62, LC3BII/I, ATG5, Beclin1 were detected by western blotting using $\beta$ - Actin as an internal control. $(n=3$; error bar, $S D)$. *Represents ${ }^{*} P<0.05,{ }^{*} \mathrm{P}<0.01$ and ${ }^{* * *} \mathrm{P}<0.001$.

(A)

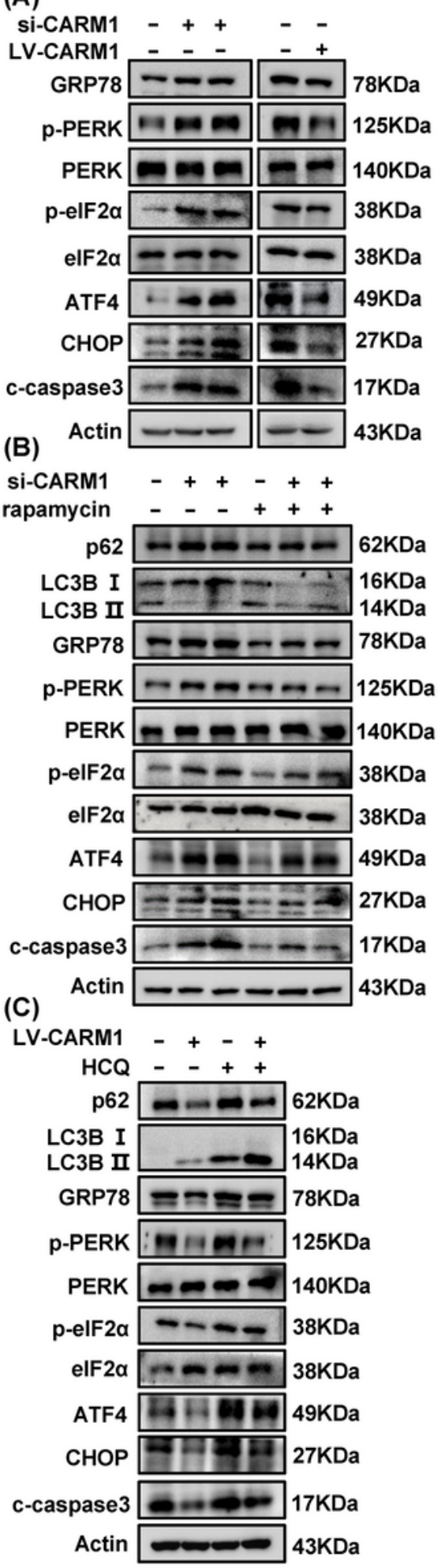

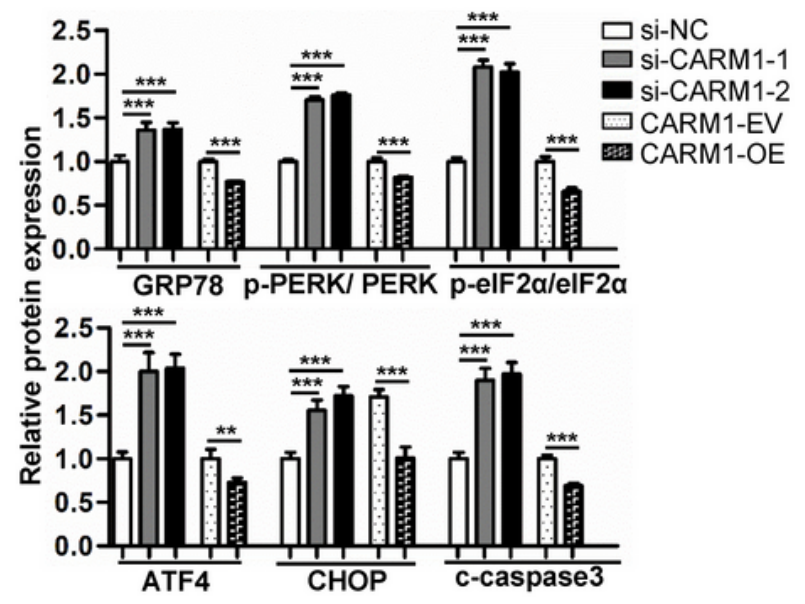
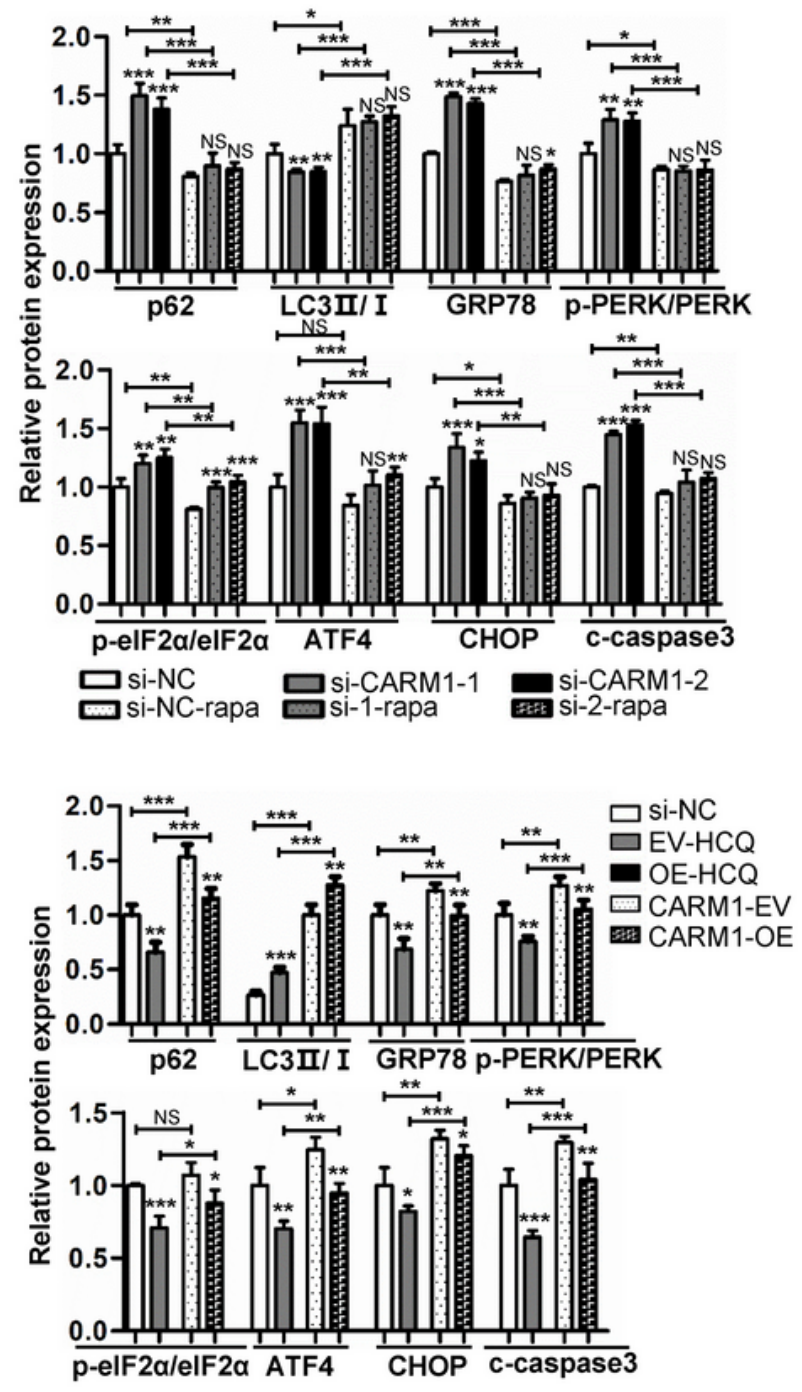

Figure 3

Blocked autophagy flux induces endoplasmic reticulum(ER) stress. A ER stress related proteins GRP78, pPERK, PERK, p-eif2a, eif2a, ATF4 and downstream effector proteins associated with apoptosis were 
detected by western blotting in CARM1 downregulation HGC27 cells and CARM1 upregulation BGC823 cells. B HGC27 CARM1-knockdown and control cells were treated with rapamycin (50nM, 24h) to detect the effect of autophagy induction on ER stress. C BGC823 overexpression and control cells were treated with HCQ $(25 \mu \mathrm{M}, 24 \mathrm{~h})$ to evaluate the role of autophagy flux blockage in ER stress. $\beta$ - Actin was applied as loading controls. Each experiment was repeated three times. Data represent mean values \pm SD. *Symbolizes * $\mathrm{P}<0.05,{ }^{* *} \mathrm{P}<0.01$ and ${ }^{* * * \mathrm{P}}<0.001$.
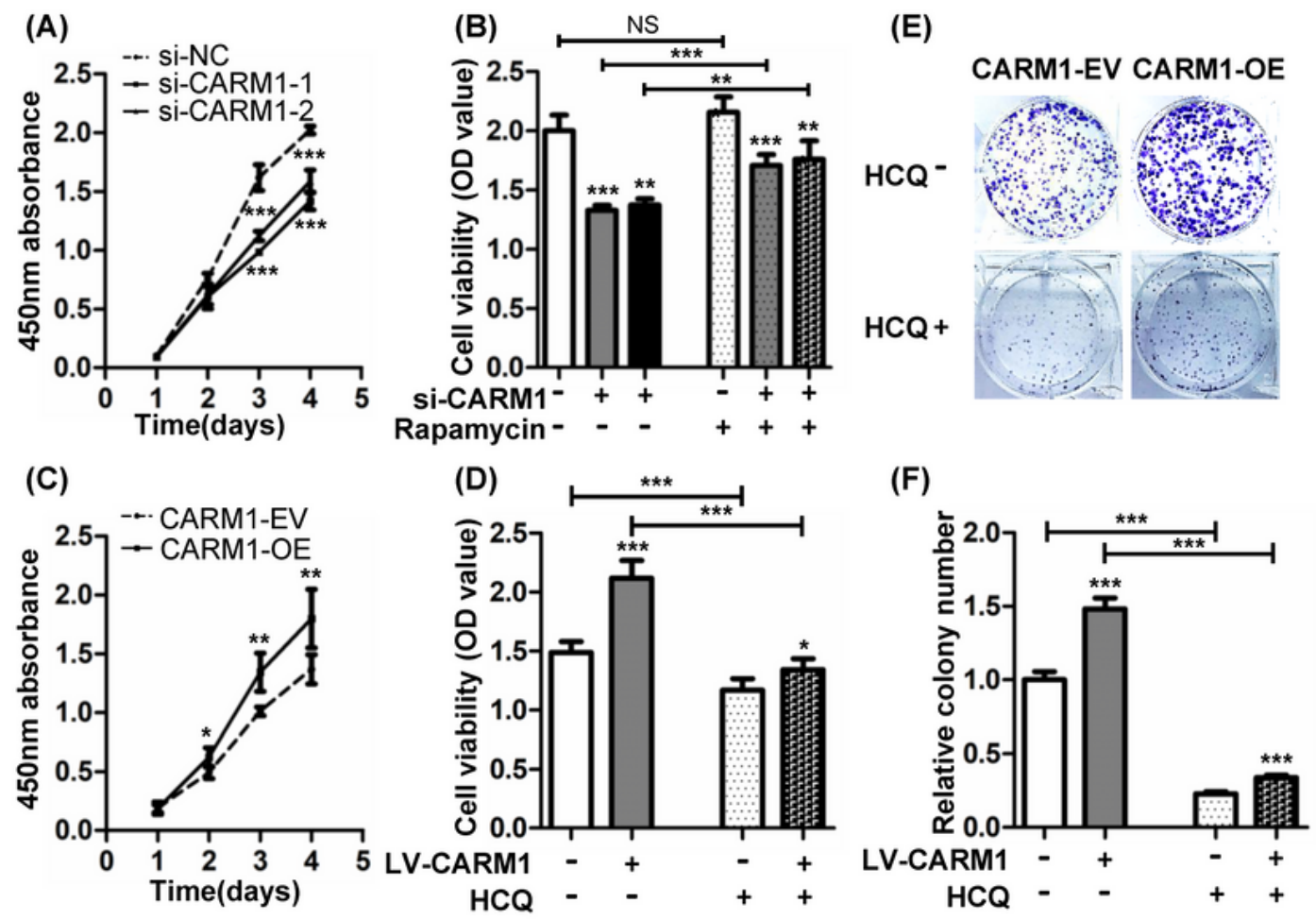

(G)

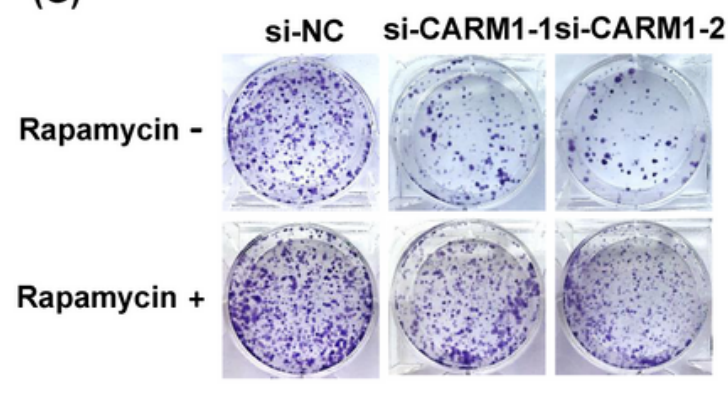

(I)

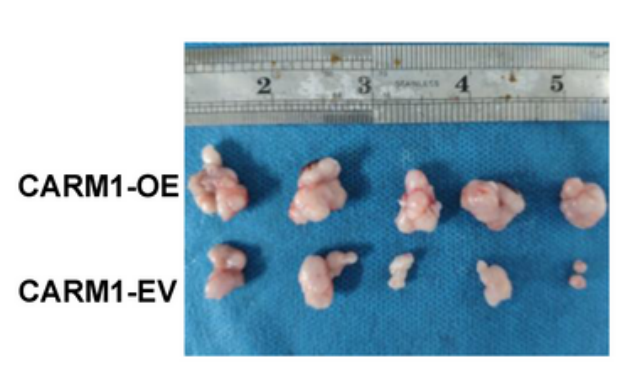

(J)

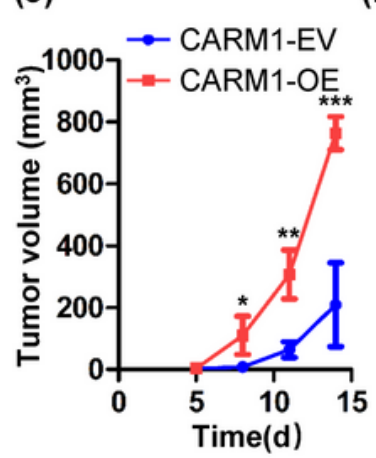

(H)

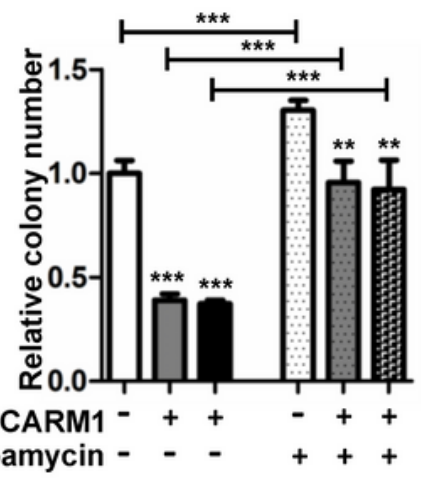

(K)

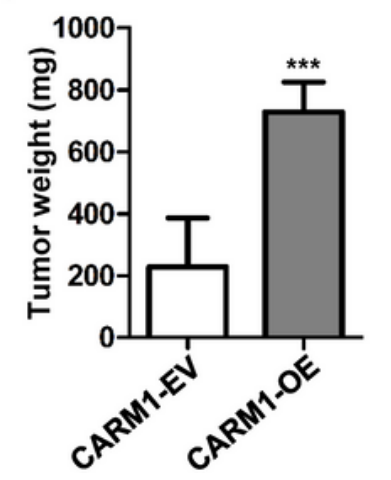

Figure 4 
CARM1 promotes the proliferation of GC cells through regulation of autophagy. A CCK-8 assay revealed that downregulation of CARM1 significantly suppressed the growth rate in HGC27 cells. B HGC27 CARM1-knockdown and control cells were treated with rapamycin $(50 \mathrm{nM}, 24 \mathrm{~h})$. Then cell viability was detected by CCK-8 assay. C CCK-8 assay proved that upregulation of CARM1 considerably enhanced cell viability in BGC823 cells. D BGC823 overexpression and control cells were treated with HCQ $(25 \mu \mathrm{M}, 24 \mathrm{~h})$. Then cell viability was determined by CCK-8 assay. E and F BGC823 stably transfected with overexpression and control lentivirus cells were treated with HCQ $(25 \mu \mathrm{M}) 3$ days later after cells were seeded. Then cells were continually cultured for 10-14 days until visible clones were formed. $\mathrm{G}$ and $\mathrm{H}$, HGC27 cells were transfected with CARM1 siRNA every 5 days to maintain knockdown effect. Cells were cultured in medium containing rapamycin (50nM) for 10-14 days until visible clones were formed. Representative images of clones were shown from three independent experiments. I Photographs of dissected xenograft tumors showed that the overexpression of CARM1 (top) also accelerated tumor growth in vivo compared to controls (bottom). $\mathrm{J}$ Tumor volumes at indicated time were evaluated following this formula: tumor volume $(\mathrm{mm} 3)=[$ length $(\mathrm{mm}) \times$ width $(\mathrm{mm}) 2] \times \pi / 6 . \mathrm{K}$ Tumor weights were also measured, indicating that the xenograft tumors derived from CARM1 overexpression cells grew faster than that injected of control gastric cells. Data are presented as mean \pm SD. *Represents $* P<0.05$, $\star \star \mathrm{P}<0.01$ and $* \star \star \mathrm{P}<0.001$. 

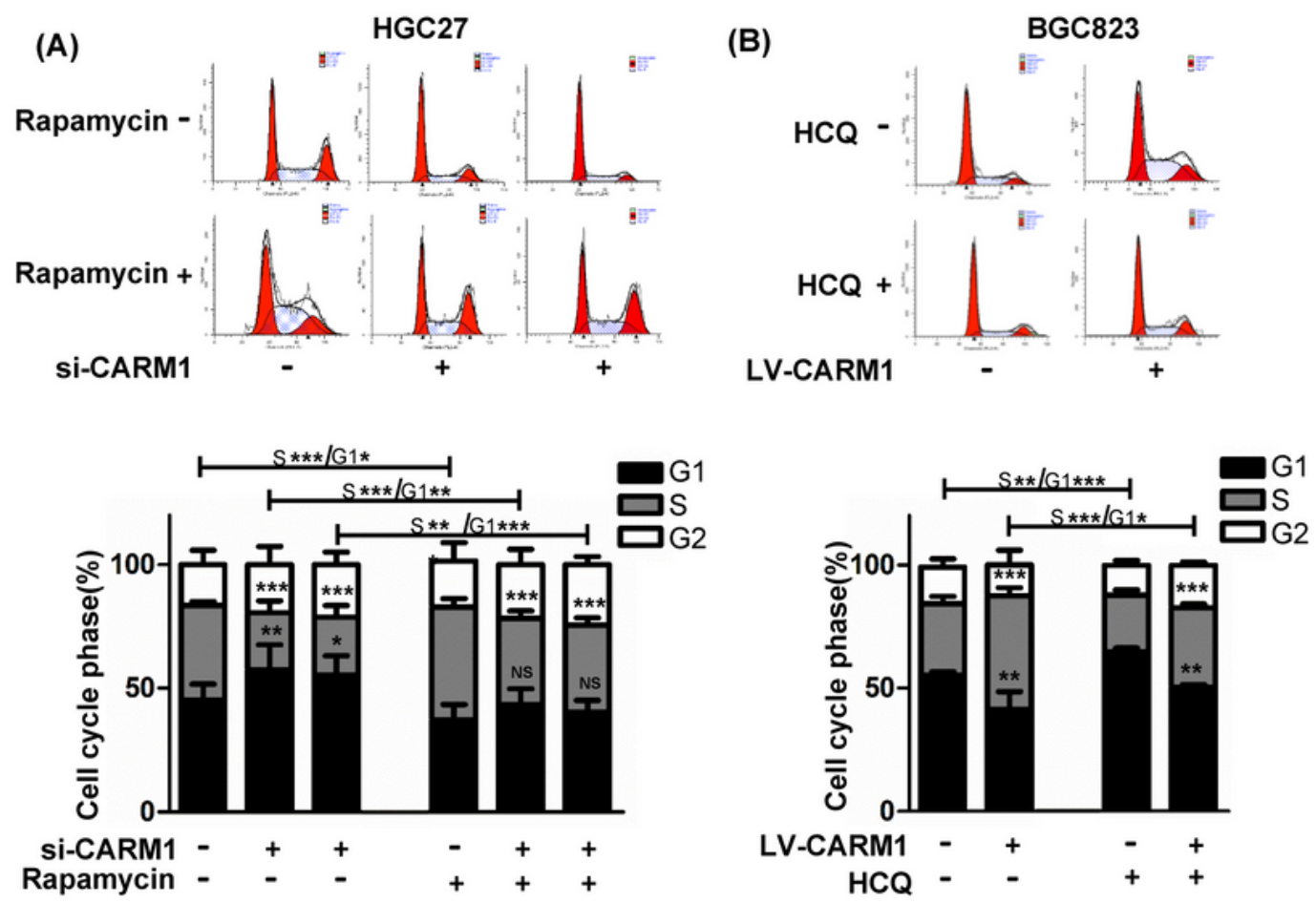

(C)

HGC27

(D)

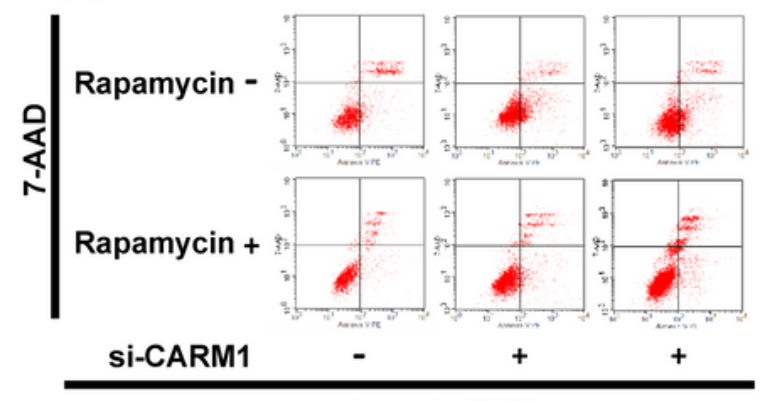

Annexin V PE
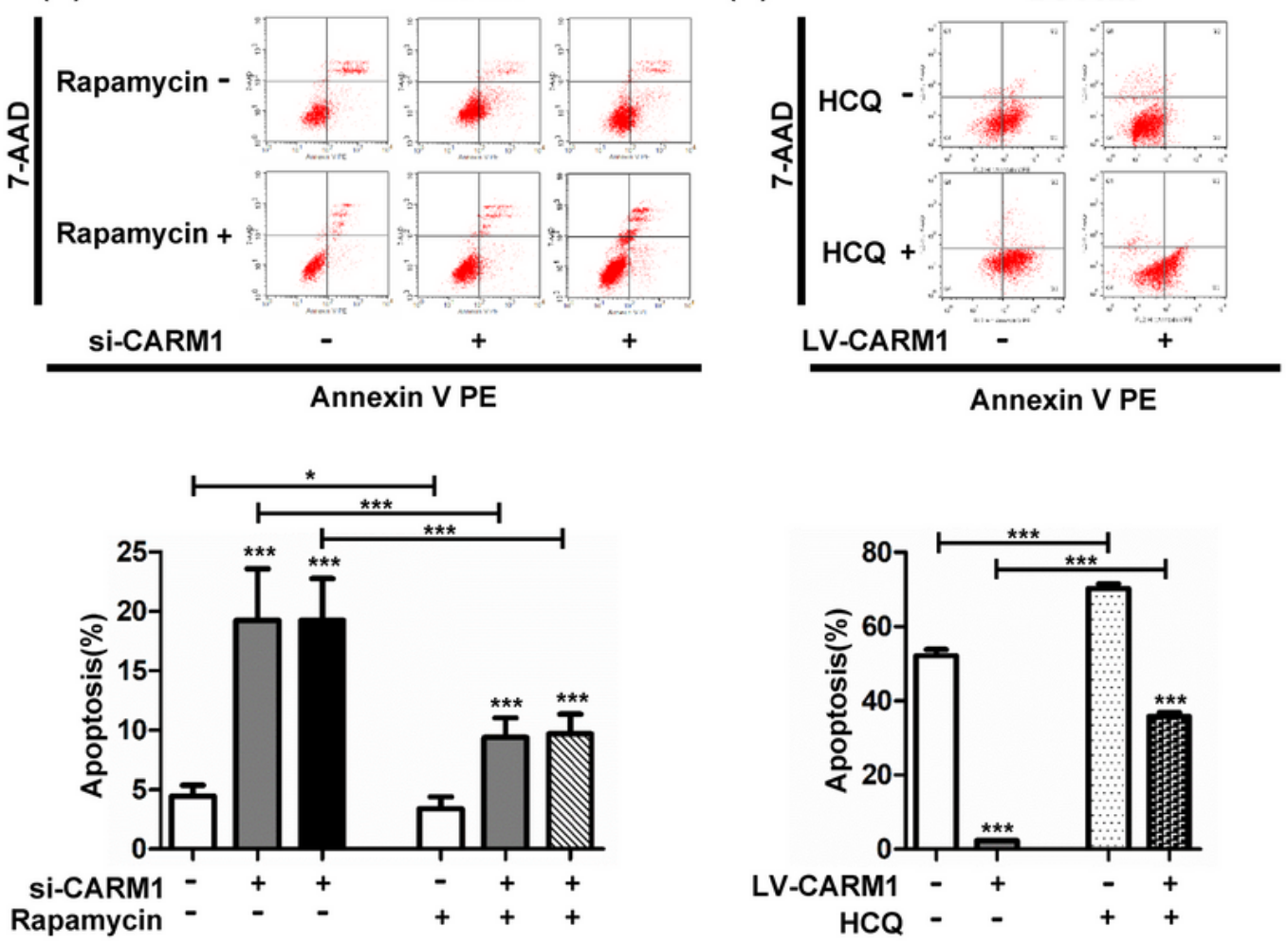

Figure 5

CARM1 potentiates cell cycle G1-S transition and inhibits apoptosis of GC cells by inducing autophagy. HGC27 cells transfected with si-CARM1 were treated with rapamycin (50nM, 24h), BGC823 cells transduced with overexpression and control lentivirus were treated with HCQ $(25 \mu \mathrm{M}, 24 \mathrm{~h})$ before the detection of flow cytometry. A and B Distribution of different cell cycle phases was presented in indicated cells by flow cytometry. CARM1 overexpression promoted while CARM1 downregulation attenuated G1-to- 
$S$ transition of GC cells. However, the effects could be reversed by autophagy activator rapamycin and autophagy inhibitor HCQ respectively. C and D Representative images showed the percentage of cells undergoing apoptosis from three dependent experiments. Treatment with rapamycin partially reversed the increase of apoptosis in CARM1 knockdown HGC27 cells (left panel), and HCQ recovered the decrease of apoptosis in CARM1 overexpression cells to some extent (right panel). Bars represent mean \pm SD from three independent experiments. *Represents $* P<0.05$, $* * P<0.01$ and ${ }^{* * *} \mathrm{P}<0.001$.

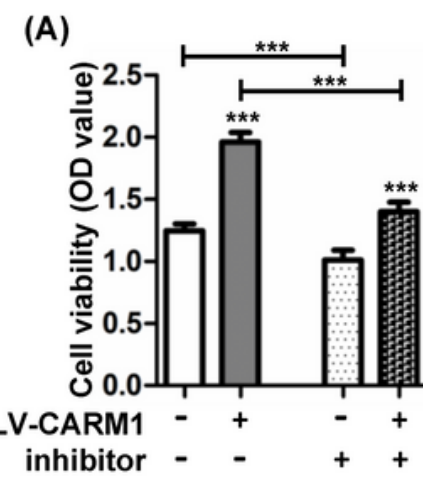

(B)

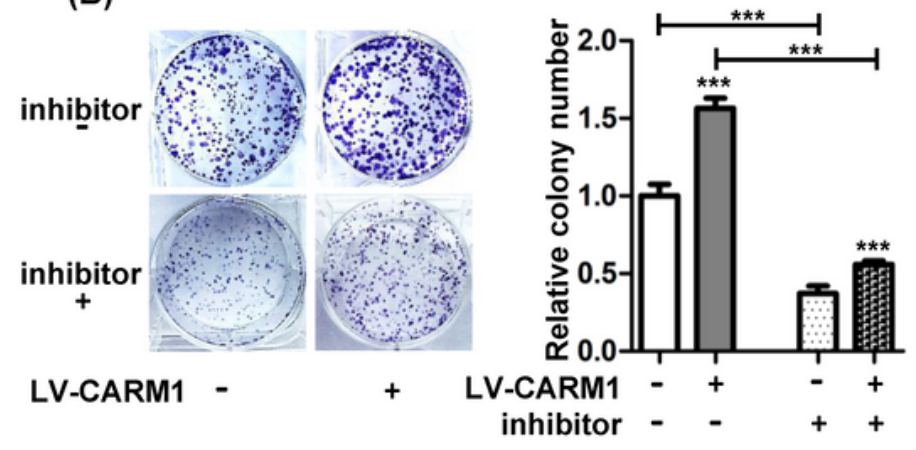

(C)

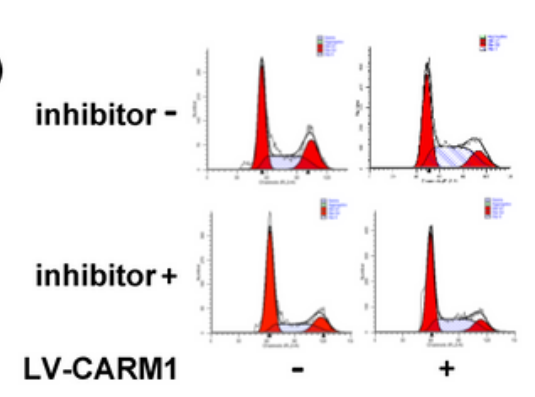

(D)

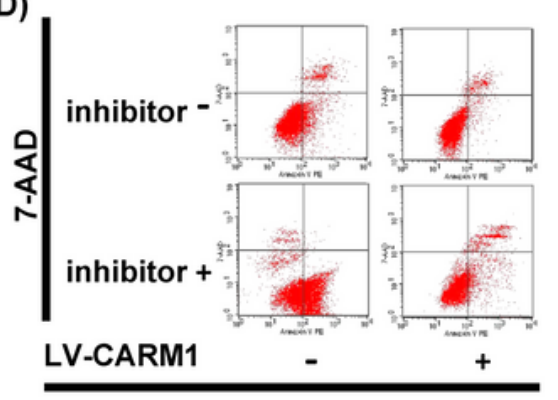

Annexin V PE

(E)

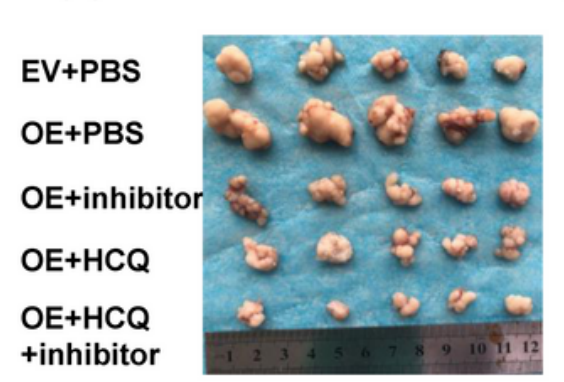

(F)
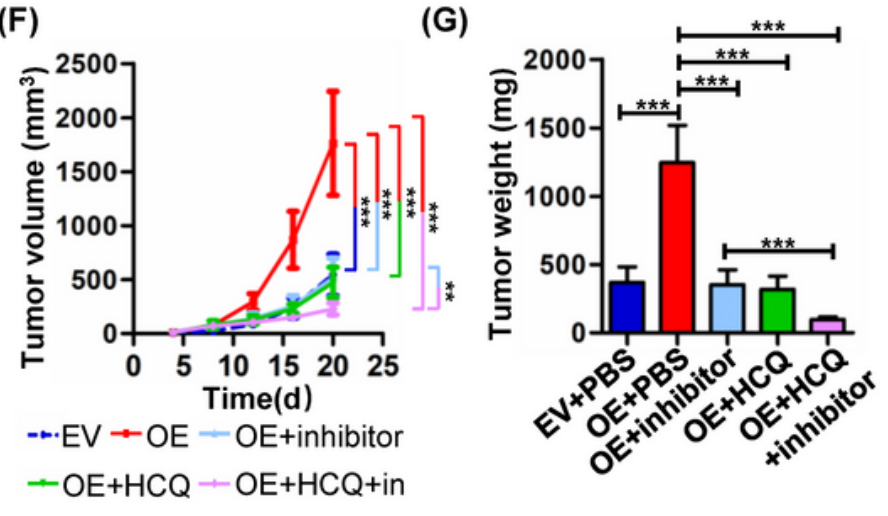

Figure 6 
CARM1 inhibitor rescued the tumor-promoting role of CARM1 both in vitro and in vivo. A BGC823 control and upregulation cells were treated with CARM $1 \mathrm{i}(8 \mu \mathrm{M}, 24 \mathrm{~h})$, the cell viability was determined by CCK-8 assay. B BGC823 control and upregulation cells were cultivated in medium containing $8 \mu \mathrm{M}$ CARM1i for 10-14 days, CARM1i reduced the increase of clone numbers caused by CARM1 overexpression. $C$ and $D$ BGC823 control and CARM1-overexpression cells were treated with CARM1i $(8 \mu \mathrm{M}, 24 \mathrm{~h})$. Cell cycle phase distribution and apoptosis in indicated were assessed by flow cytometry. E, F and G BGC823 xenograft model was performed to investigate the therapeutic effect of CARM1i and HCQ on GC in vivo. Nude mice injected with CARM 1 overexpression cells were randomly divided into four groups at day 6 when tumor volumes reached $50 \mathrm{~mm} 3$. CARM1i was performed twice daily at $100 \mathrm{mg} / \mathrm{kg}$ i.p. HCQ was administered once daily at $50 \mathrm{mg} / \mathrm{kg}$ i.p. The combination group was given two treatments and the control group was only intraperitoneally injected with PBS. E The photograph of subcutaneous tumors separated from indicated groups. $F$ The tumor volumes were measured every four days, following this formula: tumor volume $(\mathrm{mm} 3)=[$ length $(\mathrm{mm}) \times$ width $(\mathrm{mm}) 2] \times \pi / 6 . G$ The tumor weights were evaluated. Both CARM1 $\mathrm{i}$ and HCQ could slow down the tumor growth of GC. Furthermore, CARM1i exhibited a synergistic effect in combination with the treatment of HCQ. Data are demonstrated as mean $\pm \mathrm{SD}$. ${ }^{*}$ Represents $* \mathrm{P}<0.05$, $* * \mathrm{P}$ $<0.01$ and $* * * P<0.001$. 
(A)

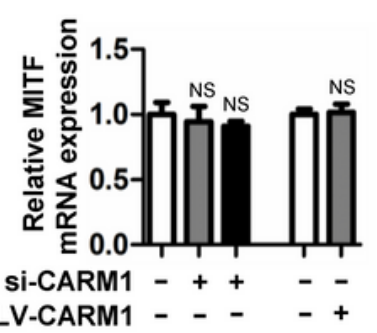

(B)

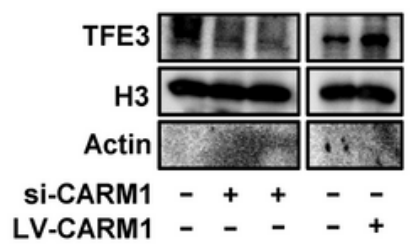

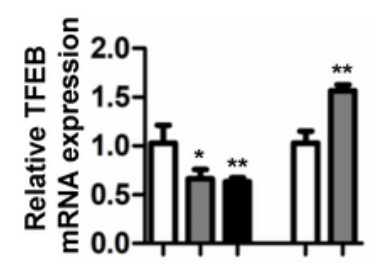

Si-CARM1 - + + - -

LV-CARM1 - - - - +

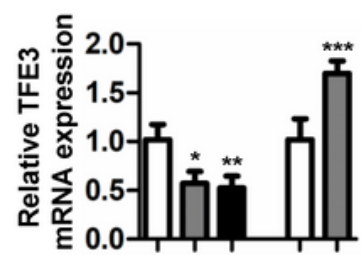

si-CARM1 - + + - -

LV-CARM1 - - - +
(D)

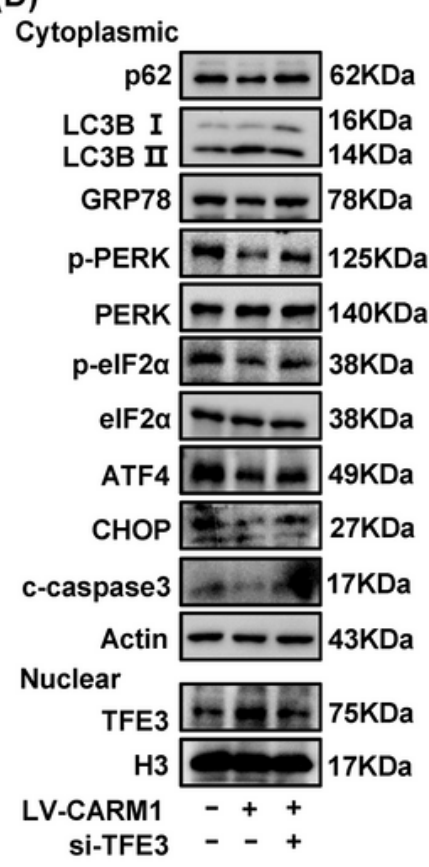

(C)

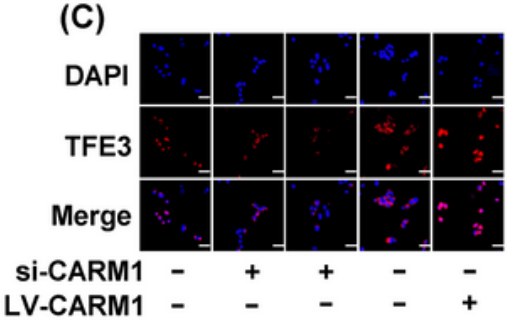

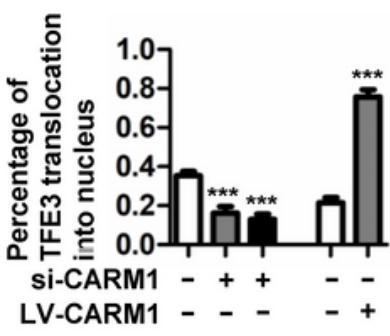

(E)

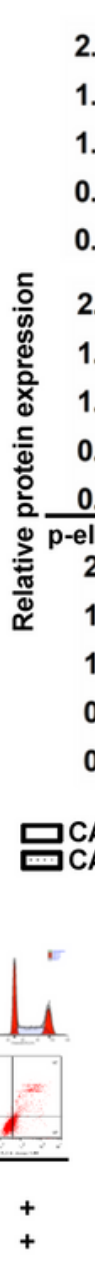

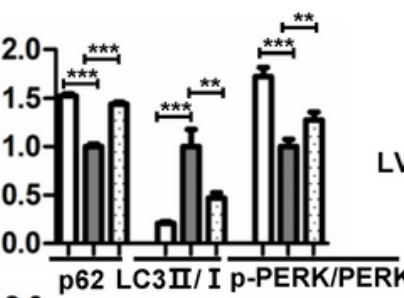

LV-CARM1 Si-TFE3 -

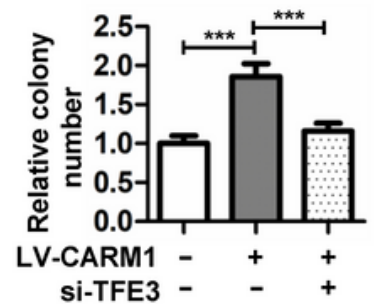

(F)

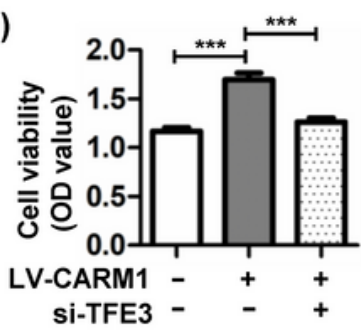

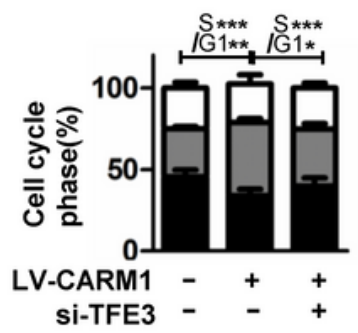

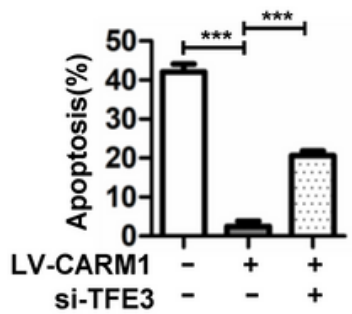

(G)

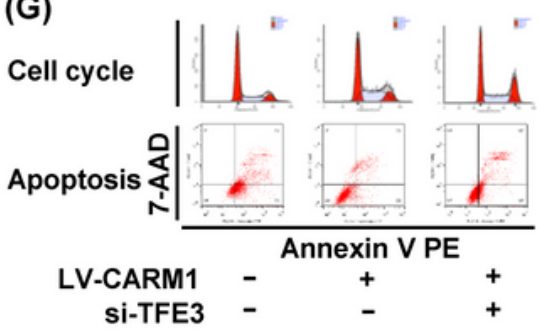

Figure 7

CARM1 activates autophagy by promoting TFE3 nuclear translocation. A The mRNA expression of MiTF/TFE family (TFE3, TFEB, MITF) was detected by qRT-PCR in CARM1 knockdown HGC27 cells and CARM1 overexpression BGC823 cells. B Western blotting assessed TFE3 nuclear protein levels in CARM1 treated cells, histone H3 was used as a loading control of nuclear protein. C The percentage of TFE3

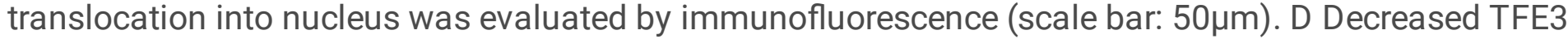


expression in BGC823 overexpression cells blocked autophagy flux and subsequently induced ER stress. Autophagy and ER stress related protein expression was detected in the cytoplasm and TFE3 expression was proved in nucleus protein by western blotting. $\beta$ - Actin and $\mathrm{H} 3$ served as loading controls for cytoplasmic proteins and nuclear proteins respectively. $\mathrm{E}$ and $\mathrm{F}$ Colony formation and CCK-8 assays revealed TFE3 downregulation reversed the proproliferative effect of CARM1 on BGC823 cells. G TFE3 knockdown rescued increased G1-S transition and decreased apoptosis resulted from CARM1 overexpression in BGC823 cells. Bars represent mean \pm SD from three independent experiments. *Represents ${ }^{*} \mathrm{P}<0.05,{ }^{*} \mathrm{P}<0.01$ and ${ }^{* *} \mathrm{P}<0.001$. 
(A)

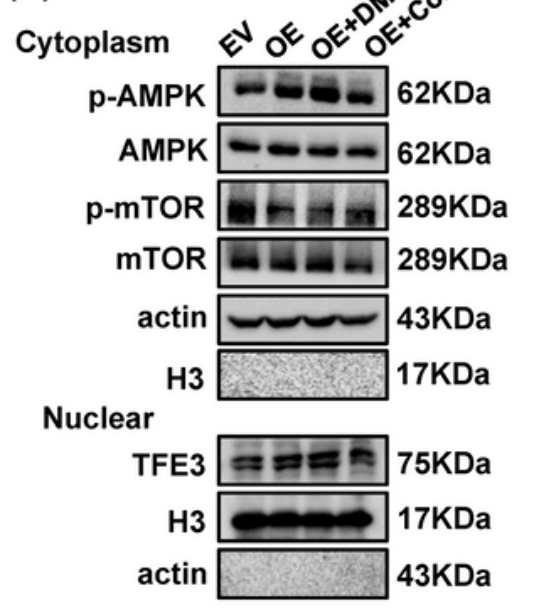

(B)

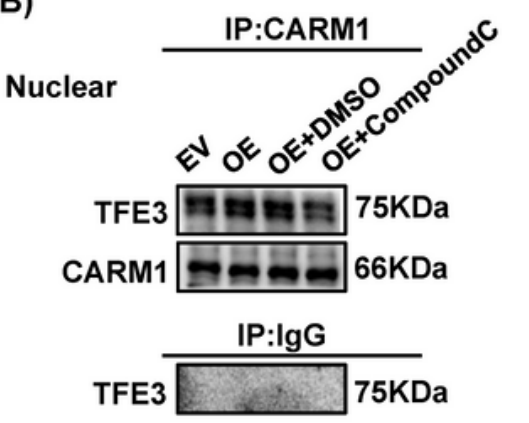

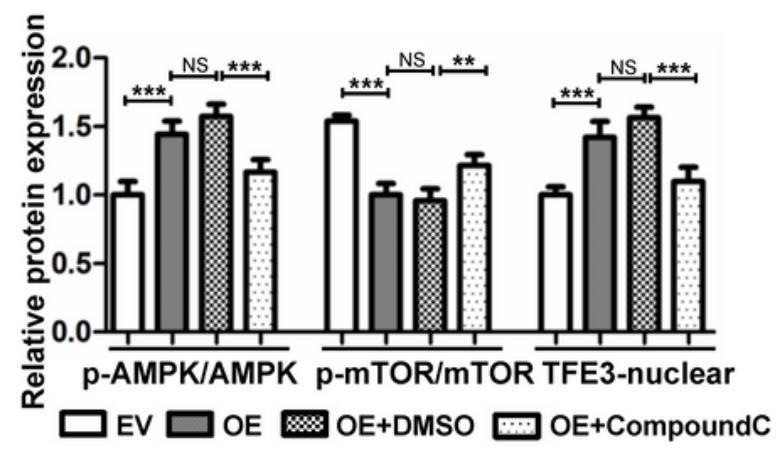

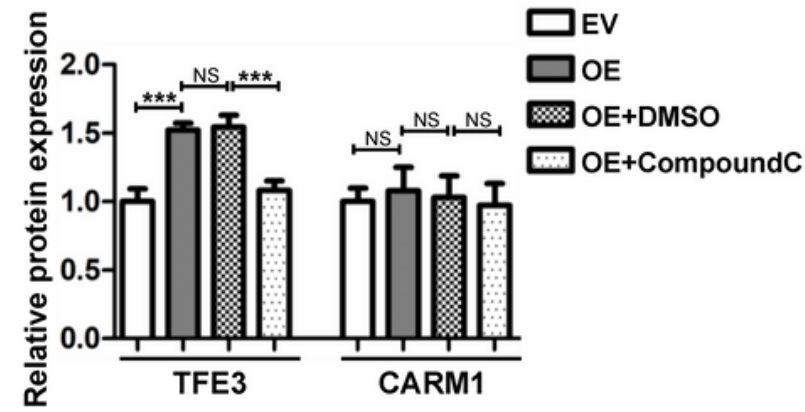

(C)

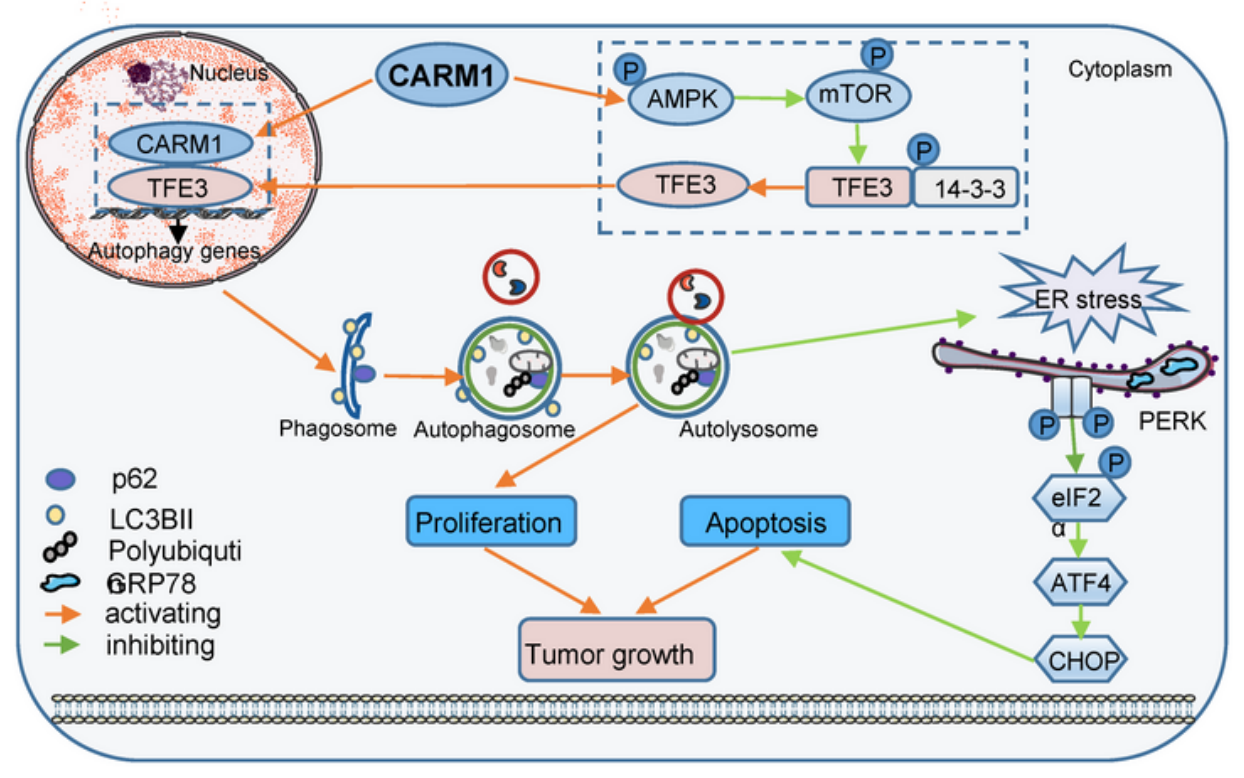

\section{Figure 8}

The TFE3 activity is activated via cytoplasmic AMPK-mTOR and nuclear CARM1-TFE3 signaling pathways. A Western blotting analysis of AMPK-mTOR signal pathway in the cytoplasm and TFE3 expression in the nucleus in CARM1-EV, CARM1-OE, CARM1-OE+DMSO, CARM1-OE+Compound C (10 $\mu M$, $6 \mathrm{~h})$ groups. B BGC823 overexpression cells were treated with Compound C $(10 \mu \mathrm{M}, 6 \mathrm{~h})$ or solvent comparison DMSO. Then immunoprecipitation assay using CARM1 antibody was performed and western 
blotting demonstrated the amount of TFE3 protein bound by CARM1 in different groups. Values represent means $\pm S D$. ${ }^{\star *} P<0.01$ and ${ }^{* \star *} P<0.001$. C Schematic model for the mechanisms of CARM1 in GC. CARM1 potentiated autophagy by facilitating TFE3 nuclear translocation and activity via activating cytosol AMPK-mTOR and nuclear CARM1-TFE3 signaling pathways. Then CARM1 promoted GC cell proliferation and reduced ER stress-induced apoptosis by regulating autophagy.

\section{Supplementary Files}

This is a list of supplementary files associated with this preprint. Click to download.

- Additionalfile2legendofFig.S1.docx

- Additionalfile3Tables1.docx

- Additionalfile4TableS2.docx

- Additionalfile5Tables3.docx

- Additonalfile1Fig.S1.tiff

- Originaldata.pdf 